\title{
Lateral surface non-uniformities in drying latex films
}

\author{
Venkata R. Gundabala ${ }^{1}$, Chun-Hong Lei $^{2}$, Keltoum Ouzineb ${ }^{3}$, Olivier Dupont ${ }^{3}$, \\ Joseph L. Keddie ${ }^{2}$, and Alexander F. Routh ${ }^{1, *}$ \\ 1. Department of Chemical Engineering and BP Institute, University of \\ Cambridge, Pembroke Street, Cambridge, CB2 3RA, UK \\ 2. Department of Physics, University of Surrey, Guildford, Surrey, GU2 \\ 7XH, UK
}

3. Cytec Surface Specialties, Anderlecht Str. 33, B-1620 Drogenbos, Belgium

* Corresponding author

email: afr10@cam.ac.uk

\begin{abstract}
$\underline{\text { Abstract }}$
The length scales of film thickness non-uniformities, commonly observed in polymer colloid (i.e. latex) films, are predicted. This prediction is achieved by investigating the stability behaviour of drying latex films. A linear stability analysis is performed on a base solution representing a uniformly drying latex film containing a surfactant. The analysis identifies film thickness non-uniformities over two length scales: long (millimetre) range (from lubrication theory) and short (micrometer) range (from nonlubrication theory). Evaporation and surfactant desorption into the bulk film are identified as the primary destabilizing mechanisms during drying. Experimental evidence through direct visualization and atomic force microscopy confirm the existence of non-uniformities over both length scales, which are shown to be functions of parameters such as initial particle volume fraction, surfactant amount and desorption strength, whilst being independent of drying rate.
\end{abstract}




\section{Introduction}

Growing concerns over the polluting effects of solvent-based polymer coatings has warranted the greater use of their alternatives, such as waterborne coatings cast from colloidal dispersions or latex [1]. These dispersions consist of polymer particles in a suspending medium of water. A latex, when cast onto a substrate, forms a continuous film upon drying [2]. Surfactants are usually used during the synthesis to provide colloidal stability and to hinder rapid aggregation. The surfactant distributes itself between the particle surface, bulk serum, and the air-water interface according to equilibrium adsorption relations [3]. Initially, when a latex is cast onto a substrate, the majority of the surfactant is present either on the particles or in the bulk serum, with minor amounts present at the air-water interface [4]. As drying proceeds and the surfactant concentration in the serum increases, there is greater adsorption onto the particle surfaces. An earlier model predicting the vertical surfactant distribution in latex films [5] has shown that this increasing adsorption leads to surfactant accumulation at the film surface (i.e. the air-water interface). This excess was previously cited as the cause of poor gloss and tackiness of dried films [6]. The direct cause of poor gloss is the existence of non-uniformities in film thickness, and this in turn, is due to the presence of surfactant. This phenomenon is the subject of study in this paper.

Lateral non-uniformities in water concentration in drying latex films are commonly observed and have been previously modeled [7]. Convection is a primary mechanism for surfactant transport [8], and hence non-uniformities in drying lead to lateral inhomogeneities in the surfactant distribution within the film. The existence of lateral surfactant inhomogeneities at the film surface has been confirmed through atomic force microscopy [9] and optical imaging [10]. These surfactant concentration gradients lead to surface tension gradients. The resulting stress imbalance creates a net flow in the direction of increasing surface tension, leading to thickness nonuniformities. Experiments [11-12] have provided evidence to the existence of variations in film thickness, created due to the surfactant concentration gradients at the film surface. When latex films with surfactant islands at their surfaces were 
washed with water, the surfactant disappeared but left permanent indentations on the film surface.

In a previous publication [13], we investigated the influence of surfactant on the evolution of thickness of a latex film during the drying process. The surfactant spreading was spurred by an initial gradient in surface concentration. This was created by placing a surfactant drop on the surface of a drying film. The analysis showed the effect of the initial particle volume fraction and the drying rate on the sizes of the resulting non-uniformities. During the drying of most real films, however, surfactant concentration gradients at the air-water interface are unknown and hence our previous analysis does not provide the natural length-scales of non-uniformities created during drying. In the present work, we use a linear stability analysis to predict the lengthscales associated with the drying of surfactant containing latex films.

Linear stability approaches have been widely employed in studying instabilities in thin liquid films. The fingering instability (formation of digitated structures) observed during the spreading of a surfactant drop on a thin liquid film [14] was attributed to the presence of Marangoni stresses [15]. Matar et al [16] investigated the stability of a system involving Marangoni spreading of a surfactant monolayer on a thin liquid layer in the quasi-steady state, but found the base flow to be stable for all wavenumbers. A transient mode analysis of the same system [17] however predicted amplification of disturbances. Further work using the transient analysis [18], confirmed the destabilizing influence of Marangoni stresses and also showed how the inclusion of van der Waals forces amplified the disturbances. An analysis of the rupture mechanism of tear films $[19,20]$ showed the destabilizing influence of van der Waals forces and the stabilizing effect of Marangoni stresses. Capillary forces and surface surfactant diffusivity act to stabilize the films from rupture [21].

Stability analysis studies on evaporating liquid films [22] showed that solvent mass loss due to evaporation causes a destabilizing effect, and the critical thickness at the onset of film rupture, increases with increasing evaporation rates. Warner et al [23] used a linear stability analysis to show the formation of "bands" or "rings" of nanoparticles in ultrathin films. In this case, evaporation seemed to have a significant 
effect on the distribution of particles. Fingering phenomena studies using both, a transient growth analysis and a linear stability analysis in the quasi-steady-state approximation [24], showed the perturbation growth rates to be decreasing with time due to relaxation of surfactant concentration gradients.

In this paper, we study the stability of drying latex films containing surfactants, with the aim of determining the length-scales of film thickness non-uniformities observed experimentally. The essence of the theoretical approach involves perturbing the base solution infinitesimally and performing a linear stability analysis to determine the perturbation growth rates for a combination of operating parameters. The wavelength corresponding to the fastest growing mode determines the dominant length-scale. The main difference here from previous thin film studies is accounting for evaporation, particle volume-fraction-dependent viscosity, and particles in the evolution equations.

In the present work, we perform a linear stability analysis within the quasi-steadystate approximation, on a base solution representing a uniformly drying latex film containing a surfactant. By implementing the stability analysis within the lubrication limit (thin film approximation) as well as in the non-lubrication regime, we identify two length scales of surface non-uniformities. Experimental evidence for the existence of two such length scales is provided. We show how allowing for desorption of the adsorbed surfactant (at the air-water interface) back into the bulk serum provides a conditional instability to the system. The growth rate of the disturbance decreases with time due to the stabilizing effect of viscosity. Hence, the early time solutions dominate and the length-scales of film thickness non-uniformities are drying-rate independent. The analysis shows the effect of parameters, such as initial particle volume fraction and the desorption strengths, on the non-uniformity length scales.

The rest of the paper is organized as follows. Section 2 has the governing equations and stability analysis for the lubrication theory. Section 3 provides experimental evidence for the length scales predicted from the lubrication theory. Section 4 lays out the governing equations and the details of the stability analysis in the non-lubrication limit. Section 5 reports an experimental test of the predictions from the nonlubrication theory, and some concluding remarks are provided in section 6 . 


\section{Lubrication theory}

\subsection{Governing equations}

The system considered here is a latex dispersion with a particle volume fraction of $\phi$ and viscosity $\mu$ that is cast onto a flat substrate and allowed to dry. As the surfactant is usually added during synthesis, it is assumed to be initially uniformly distributed. Drying occurs at a constant rate, giving a fixed velocity $E$ to the air-water interface. The governing equations for film height, particle volume fraction, and surfactant concentration are solved in Cartesian co-ordinates. The equations are nondimensionalized by using scaling parameters that are similar to the ones used by Gaver and Grotberg [25]:

$$
\begin{array}{llll}
\bar{x}=\frac{x}{L_{0}}, & \bar{z}=\frac{z}{H_{0}}, & \bar{\sigma}=\frac{\sigma-\sigma_{m}}{S}, & \bar{u}=\frac{u}{\left(\frac{S H_{0}}{\mu_{0} L_{0}}\right)}, \\
\bar{v}=\frac{v}{\varepsilon\left(\frac{S H_{0}}{\mu_{0} L_{0}}\right)}, & \bar{t}=\frac{t}{\left(\frac{\mu_{0} L_{0}^{2}}{S H_{0}}\right)}, & \bar{P}=\frac{P}{S / H_{0}}, & \bar{\Gamma}=\frac{\Gamma}{\Gamma_{m}}
\end{array}
$$

Dimensionless variables are represented with over-bars. The horizontal length scale $L_{0}$ is undetermined and the value is set later. The surface surfactant concentration $\Gamma$ is scaled with the saturation monolayer concentration $\Gamma_{m} . x$ is the horizontal coordinate, $z$ is the vertical co-ordinate, and $H_{0}$ is the initial film thickness. $\sigma$ is the latex dispersion surface tension and $\sigma_{m}$ is the dispersion surface tension with saturated monolayer concentration of surfactant. $S=\sigma_{0}-\sigma_{m}$, is the spreading coefficient, where $\sigma_{0}$ is the surface tension of a surfactant-free latex dispersion. $u$ and $v$ are the horizontal and vertical components of fluid velocity. The Marangoni velocity, obtained by balancing the Marangoni and viscous forces, scales the horizontal velocity. $\varepsilon=H_{0} / L_{0}$ is the ratio of length scales and is assumed to be small. Time $t$ is scaled with the Marangoni time scale and $P$ is the fluid pressure. 
The viscosity $\mu$ of the latex dispersion is related to the particle volume fraction $\phi$ through the Krieger-Dougherty relation [26]

$$
\mu=\mu_{0} \frac{\phi_{m}{ }^{2}}{\left(\phi_{m}-\phi\right)^{2}}=\mu_{0} F(\phi)
$$

where $\mu_{0}$ is the pure solvent viscosity and $\phi_{m}$ is the particle volume fraction when the particles achieve a random packing structure at the end of the drying stage.

Within the lubrication approximation, combining the Navier-Stokes equation in the absence of inertia and gravity with the continuity equation, and satisfying the boundary conditions (tangential stress balance at the air-film interface and no-slip condition at the substrate) gives the expression for horizontal velocity as

$$
\bar{u}=\frac{1}{F(\phi)}\left(\frac{\partial \bar{P}}{\partial \bar{x}}\left(\frac{\bar{z}^{2}}{2}-\bar{z} \bar{H}\right)+\frac{\partial \bar{\sigma}}{\partial \bar{\Gamma}} \frac{\partial \bar{\Gamma}}{\partial \bar{x}} \bar{z}\right)
$$

The derivation of Equation 2 from first principles is given in an earlier publication [13] and is not elaborated here. The expression for pressure in Equation 2 is obtained from the normal stress balance at the air-film interface:

$$
\bar{P}=-\left(\frac{\sigma_{m}}{S} \varepsilon^{2}\right)\left(\frac{\partial^{2} \bar{H}}{\partial \bar{x}^{2}}\right)
$$

The term $\frac{\sigma_{m}}{S} \varepsilon^{2}$, henceforth represented as $\alpha_{1}$, is indicative of the capillary strength. The vertical component of the velocity field is obtained by substituting the horizontal velocity expression (Eq 2 ) into the continuity equation:

$$
\bar{v}=\frac{\partial}{\partial \bar{x}}\left(\frac{1}{F(\phi)}\left(\frac{\partial \bar{P}}{\partial \bar{x}}\left(\frac{\bar{z}^{2}}{2} \bar{H}-\frac{\bar{z}}{6}\right)-\frac{\partial \bar{\sigma}}{\partial \bar{\Gamma}} \frac{\partial \bar{\Gamma}}{\partial \bar{x}} \frac{-2}{2}\right)\right)
$$

For an interface moving at a constant velocity $E$ due to evaporation, the kinematic boundary condition reads as

$$
\frac{\partial \bar{H}}{\partial \bar{t}}=\left.\bar{v}\right|_{\bar{z}=\bar{H}}-\left.\bar{u}\right|_{\bar{z}=\bar{H}} \frac{\partial \bar{H}}{\partial \bar{x}}-\frac{E \mu_{0}}{S \varepsilon^{2}}
$$

Substituting for the horizontal and vertical velocities at the interface (Eq 2 and Eq 4) into the kinematic boundary condition gives the evolution equation for film height 


$$
\frac{\partial \bar{H}}{\partial \bar{t}}=\frac{\partial}{\partial \bar{x}}\left(\frac{1}{F(\phi)}\left(\frac{\partial \bar{P}}{\partial \bar{x}} \frac{\bar{H}^{3}}{3}-\frac{\partial \bar{\sigma}}{\partial \bar{\Gamma}} \frac{\partial \bar{\Gamma}}{\partial \bar{x}} \frac{\bar{H}^{2}}{2}\right)\right)-\alpha
$$

where $\alpha=\frac{E \mu_{0}}{S \varepsilon^{2}}$ from Eq 5 is the time for evaporation scaled by the Marangoni time.

The particle volume fraction evolution equation is obtained from the particle conservation equation in the limit of zero particle Peclet number in the vertical direction by vertically averaging the particle conservation equation (an approach similar to the one in reference [7],

$$
\frac{\partial(\bar{H} \phi)}{\partial \bar{t}}=\frac{\partial}{\partial \bar{x}}\left(\frac{\bar{x}}{F(\phi)} \phi\left(\frac{\partial \bar{P}}{\partial \bar{x}} \frac{\bar{H}^{3}}{3}-\frac{\partial \bar{\sigma}}{\partial \bar{\Gamma}} \frac{\partial \bar{\Gamma}}{\partial \bar{x}} \frac{\bar{H}^{2}}{2}\right)\right)
$$

The evolution equation for surface surfactant concentration accounts for surface diffusion, Marangoni convective transport, and desorption of excess surfactant into the bulk serum. In our earlier publication, the surfactant was assumed to be insoluble, or equivalently that drying is fast enough for surfactant desorption into bulk serum to be negligible. It was assumed that $\tau_{D}>>\tau_{d r y}$, where $\tau_{D}$ and $\tau_{d r y}$ are the desorption and drying time scales, respectively. Here we consider the case where $\tau_{d r y}$ is long enough for surfactant desorption into the serum to be important. Additionally it is assumed that surfactant adsorption is much faster than the desorption. This results in a term for continuous desorption in the evolution equation [27]:

$$
\frac{\partial \bar{\Gamma}}{\partial \bar{t}}=\frac{1}{P e_{S}} \frac{\partial^{2} \bar{\Gamma}}{\partial \bar{x}^{2}}-\frac{\partial}{\partial \bar{x}}\left(\frac{1}{F(\phi)}\left(-\frac{\bar{H}^{2}}{2} \frac{\partial \bar{P}}{\partial \bar{x}} \bar{\Gamma}+\frac{\partial \bar{\sigma}}{\partial \bar{\Gamma}} \frac{\partial \bar{\Gamma}}{\partial \bar{x}} \bar{H} \bar{\Gamma}\right)\right)-\alpha_{2}\left(\bar{\Gamma}-\bar{\Gamma}_{s}\right) \bar{H}
$$

$P e_{s}=\frac{S H_{0}}{\mu_{0} D_{S}}$ is the surface diffusive Peclet number, giving the ratio of convection to surface diffusion. $D_{S}$ is the surface diffusivity of the surfactant. $\alpha_{2}$ is the ratio of Marangoni and desorption time scales giving the strength of desorption. $\bar{\Gamma}_{s}$ is the surfactant concentration in the sub-surface that is in equilibrium with the surfactant in the bulk latex serum. The desorption term is valid when the surface and subsurface concentrations are in local equilibrium, and as discussed by Jensen and Grotberg [30] 
the desorption is dependent on the film thickness since this determines the amount of fluid available for desorption of surfactant.

The governing equations 6,7 , and 8 define the drying process of a latex dispersion containing surfactant. The next sub-section shows the details of the linear stability analysis performed on the governing equations.

\subsection{Linear Stability Analysis}

Here we perform a linear stability analysis of the evolution equations derived in the previous section to obtain a length-scale prediction for surface non-uniformities. The method involves introducing small fluctuations in the film height $\bar{H}$, particle volume fraction $\phi$, and surface surfactant concentration $\bar{\Gamma}$. Henceforth, the over-bars for the dimensionless variables are eliminated for simplicity. The evolution equations 6,7 , and 8 are linearized about base solutions for $H, \phi$, and $\Gamma$ using normal modes:

$$
\begin{gathered}
H=H_{b}+\hat{H} \exp \left(\omega_{c} t+i k x\right) \\
\phi=\phi_{b}+\hat{\phi} \exp \left(\omega_{c} t+i k x\right) \\
\Gamma=\Gamma_{b}+\hat{\Gamma} \exp \left(\omega_{c} t+i k x\right)
\end{gathered}
$$

$\omega_{c}$ is the complex growth rate and $k$ is the wavenumber of the perturbation. The terms with subscript $b$ are the base solutions and the terms with hats are the perturbation amplitudes. The base state chosen represents film thinning due to evaporation only, i.e.

$H_{b}=1-\alpha t \quad ; \quad \phi_{b}=\phi_{0} /(1-\alpha t) \quad ; \quad \Gamma_{b}=\Gamma_{0}(t)$

where $\Gamma_{0}$ is the surfactant at the air-water interface at a given time $t$. Although the base states are time dependent, it is possible to use the normal modes using the quasisteady-state-approximation. This is based on the assumption that the time scale over which the base states change is much longer than the perturbation time scale, making the base states effectively time independent. 
Substituting for perturbations from Eq 9 into the evolution equations 6, 7, and 8 and ignoring terms higher than linear in the perturbation gives a system of linear equations of the form A.X=0, where

$\mathbf{X}=\left[\begin{array}{c}\hat{H} \\ \hat{\phi} \\ \hat{\Gamma}\end{array}\right] ; \quad \mathbf{A}=\left[\begin{array}{lll}C_{11} & C_{12} & C_{13} \\ C_{21} & C_{22} & C_{23} \\ C_{31} & C_{32} & C_{33}\end{array}\right]$

Here $\mathbf{A}$ is the coefficient matrix, the elements of which are given by

$$
\begin{aligned}
& C_{11}=\phi_{m}{ }^{2} \omega+\frac{k^{4}}{3} H_{b}{ }^{3}\left(\phi_{m}-\phi_{b}\right)^{2} \alpha_{1} \\
& C_{12}=0 \quad ; \quad C_{13}=\frac{k^{2}}{2} H_{b}{ }^{2}\left(\phi_{m}-\phi_{b}\right)^{2} \\
& C_{21}=\frac{\partial \phi_{b}}{\partial t} ; \quad C_{22}=\omega H_{b}+\frac{\partial H_{b}}{\partial t} ; C_{23}=0 \\
& C_{31}=-\frac{k^{4}}{2} \alpha_{1}\left(\phi_{m}-\phi_{b}\right)^{2} H_{b}{ }^{2} \Gamma_{b}-\phi_{m}{ }^{2} \alpha_{2}\left(\Gamma_{b}-\Gamma_{S}\right) \quad ; \quad C_{32}=0 \\
& C_{33}=-\frac{k^{2}}{P e_{S}} \phi_{m}{ }^{2}-\phi_{m}{ }^{2} \omega-k^{2}\left(\phi_{m}-\phi_{b}\right)^{2} H_{b} \Gamma_{b}-\phi_{m}{ }^{2} \alpha_{2} H_{b}
\end{aligned}
$$

where $\omega$ is the real part of the complex growth rate $\omega_{c}$. For the system of equations represented by $\mathbf{A} . \mathbf{X}=0$ to have non-trivial solutions, the determinant of the coefficient matrix should be zero. This gives

$$
C_{22}\left(C_{11} C_{33}-C_{13} C_{31}\right)=0
$$

which provides the possible solutions $C_{22}=0$ or $C_{11} C_{33}-C_{13} C_{31}=0$

Setting $C_{22}=0$ gives one possible growth rate as:

$$
\omega_{1}=\frac{\alpha}{1-\alpha t}
$$

As $\alpha t<1$ for all times, Eq 11 indicates that the system is unstable (due to evaporation), although the growth rate is independent of the wavenumber $k$. Hence, no specific wavelength is selected with this mode, and so some other physics must be responsible for the observed instability. Setting $C_{11} C_{33}-C_{13} C_{31}=0$ yields a quadratic expression in $\omega$, of the form

$$
\begin{gathered}
\omega^{2}+b \omega+c=0 \\
b=\left(\alpha_{1} \frac{H_{b}{ }^{3}}{3} \frac{\left(\phi_{m}-\phi_{b}\right)^{2}}{\phi_{m}{ }^{2}}\right) k^{4}+\left(H_{b} \Gamma_{b} \frac{\left(\phi_{m}-\phi_{b}\right)^{2}}{\phi_{m}{ }^{2}}+\frac{1}{P e_{S}}\right) k^{2}+\alpha_{2} H_{b}
\end{gathered}
$$




$$
\begin{aligned}
& c=\alpha_{1}\left(\frac{H_{b}{ }^{3}}{3 P e_{S}} \frac{\left(\phi_{m}-\phi_{b}\right)^{2}}{\phi_{m}{ }^{2}}+\frac{H_{b}{ }^{4} \Gamma_{b}}{12} \frac{\left(\phi_{m}-\phi_{b}\right)^{4}}{\phi_{m}{ }^{4}}\right) k^{6}+\left(\frac{\alpha_{1} \alpha_{2}}{3} H_{b}{ }^{4} \frac{\left(\phi_{m}-\phi_{b}\right)^{2}}{\phi_{m}{ }^{2}}\right) k^{4}- \\
& \left(\frac{\alpha_{2}}{2} H_{b}{ }^{2}\left(\Gamma_{b}-\Gamma_{S}\right) \frac{\left(\phi_{m}-\phi_{b}\right)^{2}}{\phi_{m}{ }^{2}}\right) k^{2}
\end{aligned}
$$

The solution to the quadratic (Eq 12) gives the expressions for the growth rates $\omega_{2}$ and $\omega_{3}$ as

$$
\begin{aligned}
& \omega_{2}=\frac{-b-\sqrt{b^{2}-4 c}}{2}, \\
& \omega_{3}=\frac{-b+\sqrt{b^{2}-4 c}}{2}
\end{aligned}
$$

$\omega_{2}$ is always negative leaving the sign of $\omega_{3}$ to determine the evolution of the perturbation. The next sub-section shows the behaviour of the growth rate $\omega_{3}$ and the corresponding variation in the non-uniformity length scale under various drying conditions.

\subsection{Theoretical predictions}

As stated in Section 1, when a latex film containing surfactant is unstable to disturbances, under certain drying conditions, the wavelength corresponding to the fastest growing mode determines the length-scale for the non-uniformity created by the instability. Under given drying conditions, the wavenumbers for which the system is unstable, are determined from the plots of growth rate $\left(\omega_{3}\right)$ against the wavenumber

$k$. The dimensionless length scale (henceforth represented by $\lambda_{N U}$ ) is then just the wavelength corresponding to the fastest growing mode. The dimensional value of the non-uniformity length (represented by $L_{N U}$ ) is given by $L_{N U}=\lambda_{N U} L_{0}$. The horizontal length scale $L_{0}$ is obtained by fixing one of the "free parameters" of the system (Eq 13). Before discussing the stability analysis results, for the purpose of clarity, here we define explicitly the set of "free parameters" involved in the system: $\alpha=\frac{E \mu_{0} L_{0}{ }^{2}}{S H_{0}{ }^{2}}$ is the ratio of Marangoni to evaporative time scales; 
$\alpha_{1}=\frac{\sigma_{m} H_{0}{ }^{2}}{S L_{0}{ }^{2}}$ is the ratio of capillary to Marangoni driving forces;

$\alpha_{2}=\frac{D_{b} \mu_{0} L_{0}{ }^{2}}{S}$ is the ratio of Marangoni to desorption time scales ( $D_{b}$ is the desorption coefficient); and

$P e_{s}=\frac{S H_{0}}{\mu_{0} D_{S}}$ is the ratio of Marangoni convection to surface diffusion strengths.

The desorption coefficient, $D_{b}$, can be seen as a ratio of, the surfactant flux from the film surface into the bulk, to the concentration difference between the surface and the bulk. The continuous desorption from the surface can be considered to be a result of particle compaction close to the top surface acting as a surfactant source during the film drying.

The value of any one of these "free parameters" can be fixed to determine the horizontal length scale $L_{0}$. Here we fix $\alpha_{1}$ at an arbitrary value and evaluate the rest of the parameters accordingly. As will be shown from Figures 1 and 2, the dimensional value of non-uniformity length $\left(L_{N U}\right)$ is independent of the value of $\alpha_{1}$ chosen. Table 1 lists two sets of parameter values used to show an example case of stability behaviour for fixed operating conditions. The operating conditions such as $E$, $H_{0}, S$ are the same for both sets of parameters and hence the dimensional nonuniformity length, $L_{N U}$, should be the same. The values from Table 1 are input into Eq 13 to obtain the coefficients $\mathrm{b}$ and $\mathrm{c}$ of the quadratic expression for $\omega$ (Eq 12). The growth rate $\omega_{3}$, obtained from $\mathrm{Eq} 14 \mathrm{~b}$, is then plotted against wavenumber $k$. As the base solution for film height, $H_{b}$, is a function of time, the stability behaviour for a given set of operating conditions ends up likewise being time-dependent. Figure 1 shows the growth rate $\omega_{3}$ as a function of wavenumber for the first set of parameters (set 1 ) at various times during the drying process. The total (scaled) drying time $\bar{t}_{d r y}$ is dependent on the drying parameter $\alpha$ and the initial particle volume fraction $\phi_{0}$ through the relation for $\phi$ given in $\mathrm{Eq} 10$

$$
\bar{t}_{d r y}=\left(1-\frac{\phi_{0}}{\phi_{m}}\right) \frac{1}{\alpha}
$$


Choosing $\phi_{m}=0.64$ (random packing volume fraction), and using the values for $\phi_{0}$ and $\alpha$ from set 1 in Table $1, \bar{t}_{d r y}$ is estimated to be $5.7 \times 10^{5}$. So the curve at $t=5 \times 10^{5}$ represents the stability behaviour just before the completion of drying. The curves in Figure 1 suggest that, at all times during drying, the system is stable to large wavenumber (or short-wave) disturbances and is unstable to small wavenumber (or long-wave) disturbances, a behaviour typical of thin liquid films [28]. It can also be noted that the growth of a disturbance of a given wavenumber is retarded as drying proceeds.

During the drying process, the viscosity of the latex dispersion increases due to the increase in particle volume fraction. The effect of viscosity is to dissipate the energy of any disturbance [29] and hence the decrease in growth rate with drying. In order to obtain a single wavelength (corresponding to the fastest growing mode) that gives us the prediction for size of non-uniformity for the given operating conditions, the growth rate $\omega_{3}$ needs to be integrated over the drying time:

$\int_{0}^{\bar{t}_{d r y}} \omega_{3} d t=\varpi(k)$

Figure 2 shows the time-integrated growth rate $\varpi(k)$, plotted against wavenumber for set 1 of parameters listed in Table 1. From this stability curve, the wavenumber corresponding to the maximum growth rate is obtained as 7.6, giving a dimensionless non-uniformity length scale value of $\lambda_{N U}=\frac{2 \pi}{7.6}=0.826$. Using the horizontal length scale $\left(L_{0}\right)$ value from set 1 in Table 1 , the size of surface non-uniformity created under the specified drying conditions is evaluated as $L_{N U}=1.13 \mathrm{~mm}$. To substantiate the requisite for $L_{N U}$ to be independent of $\alpha_{1}$ under constant operating conditions, we plot the time-integrated growth rate (Figure 3) using the set 2 of parameters in Table 1. The operating conditions are the same as set 1 with only the arbitrary value of $\alpha_{1}$ being different. Note that the value of $L_{0}$ evaluated for set 2 is an order of magnitude larger than that obtained for set 1 based on the definition of $\alpha_{1}\left(\alpha_{1}\right.$ varies as $\left.O\left(1 / L_{0}{ }^{2}\right)\right)$. The total (scaled) drying time $\bar{t}_{d r y}$ here is estimated to be $5.7 \times 10^{3}$ from Eq 15. The stability curve shown in Figure 3 is qualitatively the same as the one in Figure 
2, as we would expect due to constant operating conditions. Using the wavenumber corresponding to the maximum growth rate, the dimensionless non- uniformity length scale value is obtained and the dimensional value of the instability wavelength is found to be invariant to the "arbitrary" choice of free parameter.

Figure 4 shows the effect of initial particle volume fraction $\phi_{0}$ on the dimensionless length scales of non-uniformities $\left(\lambda_{N U}\right)$ created during drying. The operating parameters are the same as listed in set 1 (Table1), with only $\phi_{0}$ varying. The values for $\lambda_{N U}$ are obtained from the time-integrated growth rate curves corresponding to the specified initial volume fractions. Figure 4 suggests that the sizes of non-uniformities created decrease, almost linearly, with an increase in initial particle volume fraction. Higher initial volume fractions mean stronger viscous forces, which have the effect of dampening the growth rate of any disturbances and driving the growth rate maxima towards shorter wavelengths. Figure 5 gives an example of how growth rate dampens with increasing volume fraction. In order to minimize the non-uniformities in latex films, high particle volume fraction dispersions need to be used.

Figure 6 shows the effect of desorption strength on dimensionless length-scale $\lambda_{N U}$. $\alpha_{2}$ gives a measure of the desorption (from the air-water interface) strength, with higher $\alpha_{2}$ indicating stronger desorption and vice versa. The plot shows that the nonuniformity size decreases with an increase in the value of $\alpha_{2}$. A look at the stability curves for different $\alpha_{2}$ values indicates that when $\alpha_{2}$ is set to zero, the growth rate is exponentially decaying, i.e. the system is stable in the absence of desorption. Hence stronger desorption (higher $\alpha_{2}$ ) accelerates the growth rate and drives the growth rate maxima towards shorter wavelengths. The desoprtion of surfactant leads to a time dependence on the surface tension and hence introduces a mechanism for instability.

Figure 7 shows the effect of initial film thickness $\left(H_{0}\right)$ on the sizes of non-uniformities $\left(L_{N U}\right)$ created. As the initial film thickness affects all the time scales involved in the system, we examine its effect on the dimensional size of non-uniformity $\left(L_{N U}\right)$ rather the dimensionless non-uniformity length scale $\left(\lambda_{N U}\right)$. An increase in $H_{0}$ increases the 
value of $\alpha_{2}$, thereby driving the growth rate maxima towards shorter wavelengths and leading to smaller values of $\lambda_{N U}$. An increase in $H_{0}$ has the additional effect of strengthening the capillary forces relative to Marangoni forces and is reflected through the increase in the value of $L_{0}$. The net effect of $H_{0}$ is then dependent on the relative magnitudes of its effect on $\lambda_{N U}$ and $L_{0}$. As the curve in Figure 7 illustrates, $H_{0}$ 's effect on $\lambda_{N U}$ dominates over its effect on $L_{0}$, resulting in a net increase in size of non-uniformity with increasing $H_{0}$. Hence latex films with higher wet film thicknesses show larger sizes of non-uniformities compared to lower wet film thicknesses.

The total amount of surfactant present in the dispersion has also an effect on the nonuniformity length scales. $\bar{\Gamma}_{S}$ is the surfactant concentration in the sub-surface that is in equilibrium with the surfactant in the bulk serum. Figure 8 shows the effect of $\bar{\Gamma}_{S}$ on $\lambda_{N U}$. Increasing $\bar{\Gamma}_{S}$ reduces the driving force for desorption (Eq 8) and hence drives the growth rate maxima towards longer wavelengths, resulting in higher nonuniformity length scales. So the net effect of an increased surfactant amount in a latex dispersion is an increase in the size of non-uniformity. The next section presents some experimental evidence to support the predictions made by the lubrication theory.

\section{Experimental Investigation of long length scale instabilities}

One parameter that can be easily varied in laboratory conditions is the wet film thickness, $H_{0}$. Simple drying experiments were conducted to investigate the effect of $H_{0}$ on the sizes of non-uniformities observed in surfactant-containing latex films.

3.1 Materials: The material used here is a latex made from a copolymer of butyl acrylate (BA) and methyl methacrylate (MMA) with a $50 \%$ weight fraction of each monomer. The latex particles were synthesized via a semi-continuous emulsion polymerization process. The latex was dialyzed to remove the surfactants, free watersoluble polymer, and un-reacted monomer from the serum, leaving a $40 \mathrm{wt} \%$ solids content. A known amount (3 wt \% based on solid content) of a non-ionic surfactant 
(Triton X-100) was then added to the latex samples and a tiny amount of a blue dye (non surface active) was added to provide visual clarity to the otherwise transparent dry films.

3.2 Methods: Films of thicknesses varying from 100 to $600 \mu \mathrm{m}$ were cast onto glass substrates using a laboratory applicator, which helps in obtaining uniform wet film thicknesses. The films were allowed to dry at room temperature. Although the dried latexes turn transparent upon film formation, the blue dye aids visualization through the naked eye.

3.3 Experimental results: Close observation reveals that the regions close to the edges of the films have striations at the surface, with a uniform spacing between them. No such striations could be observed for surfactant-free latex films, indicating that the striations are a result of instabilities arising due to presence of a surfactant in a drying film. The presence of these striations indicates the existence of film thickness nonuniformities and the spacing between the striations correspond to the sizes of nonuniformities predicted by the lubrication theory. Figure 9 shows the effect of initial film thickness, $H_{0}$, on the spacing between the striations (or the size of the nonuniformities). As $H_{0}$ increases, the size of non-uniformity increases, as predicted by the theory. The observed non-uniformity length scale is of the same order as predicted by the lubrication theory (Figure 7). The deviation between the theoretical predictions and the experimental observation could be attributed to the undetermined desorption strength value.

\subsection{Validity of Lubrication Theory-Based Analysis: The lubrication} approximation is valid for smooth and gradual changes in the film height. The validity of this condition can only be checked $a$ posteriori and as can be seen in Figures 1, 2, 3 and 5 the maximum growth rate occurs at large wavenumbers (or short length scales). This observation contradicts the underlying assumption of the lubrication theorybased analysis, and so the specific predictions of Figures 6,7 , and 8 need to be taken with caution. Indeed, it can be argued that the lubrication analysis merely indicates the presence of an instability, and a full 2-D analysis is required to predict it. This analysis is carried out in Section 4. It should be noted, however, that there is 
experimental evidence for an instability on the millimeter size length scale, whjch is consistent with the predictions of the lubrication approximation.

So far, we have shown the existence of film thickness non-uniformities, spanning over a millimetre size range, in a dried latex film in the presence of a surfactant. The stability analysis within the lubrication approximation yielded a prediction of the correct length scale. Relaxing the lubrication approximation provides a prediction for an instability length scale spanning the $\mu \mathrm{m}$ size range. The reasons for allowing the relaxation are the limited validity of the lubrication approximation, as discussed above, as well as the experimental observation of micron-sized film non-uniformities as shown in Section 5. The details of the analysis and corresponding experimental verification are discussed in the next section.

\section{Non-lubrication theory}

\subsection{Governing equations:}

In this section, we relax the thin film approximation by assuming $\varepsilon$ to be of order 1 i.e. the horizontal and vertical length scales are comparable. Consequently, the problem needs to be solved in 2-D rather than in 1-D. The same scaling parameters used in the lubrication theory are used here to non-dimensionalize the system variables and the scaled variables are represented with over-bars. The governing equations to be used are the fully non-linear Navier-Stokes $(\mathrm{N}-\mathrm{S})$ equations together with the surfactant conservation equation at the film surface. The horizontal and vertical components of the $\mathrm{N}-\mathrm{S}$ equations are given by

$$
\begin{aligned}
& \frac{\partial \bar{u}}{\partial \bar{t}}+\bar{u} \frac{\partial \bar{u}}{\partial \bar{x}}+\bar{v} \frac{\partial \bar{u}}{\partial \bar{z}}+A \frac{\partial \bar{p}}{\partial \bar{x}}-A\left(\varepsilon^{2} \frac{\partial^{2} \bar{u}}{\partial \bar{x}^{2}}+\frac{\partial^{2} \bar{u}}{\partial \bar{z}^{2}}\right)=0 \\
& \frac{\partial \bar{v}}{\partial \bar{t}}+\bar{u} \frac{\partial \bar{v}}{\partial \bar{x}}+\bar{v} \frac{\partial \bar{v}}{\partial \bar{z}}+\frac{A}{\varepsilon^{2}} \frac{\partial \bar{p}}{\partial \bar{z}}-A\left(\varepsilon^{2} \frac{\partial^{2} \bar{v}}{\partial \bar{x}^{2}}+\frac{\partial^{2} \bar{v}}{\partial \bar{z}^{2}}\right)=0
\end{aligned}
$$


where $A=\frac{\mu_{0}{ }^{2}}{\rho_{0} S H_{0}} \frac{1}{\varepsilon^{2}}$ is a dimensionless quantity representing the ratio of viscous and Marangoni driving forces. The conservation equation for the surfactant at the film surface reads as

$$
\frac{\partial \bar{\Gamma}}{\partial \overline{\mathrm{t}}}=\frac{1}{\mathrm{Pe}_{\mathrm{S}}} \frac{\partial^{2} \bar{\Gamma}}{\partial \overline{\mathrm{x}}^{2}}-\frac{\partial}{\partial \overline{\mathrm{x}}}\left(\left.\overline{\mathrm{u}}\right|_{\overline{\mathrm{z}}=\overline{\mathrm{H}}} \bar{\Gamma}\right)-\alpha_{2}\left(\bar{\Gamma}-\bar{\Gamma}_{\mathrm{S}}\right) \overline{\mathrm{H}}
$$

The normal stress balance and the kinematic boundary condition at the air-film interface are the same as in the lubrication analysis (Eqns. 3 and 5). The tangential stress balance $\frac{\partial \bar{\sigma}}{\partial \bar{x}}=\frac{\partial \bar{u}}{\partial \bar{z}}$ along with the continuity equation $\frac{\partial \bar{u}}{\partial \bar{x}}+\frac{\partial \bar{v}}{\partial \bar{z}}=0$ completes the set of governing equations and boundary conditions needed to define the system. In the lubrication theory analysis (Figure 3), where a non-constant viscosity was used, the size of non-uniformity was shown to vary with particle volume fraction (and hence viscosity), albeit by a small factor. Here, in the non-lubrication theory, we use a constant viscosity in order to avoid the mathematical complications, therefore allowing an analytical solution. It should also be noted that the particle conservation equation (similar to Eq. 7) is excluded from the set of governing equations used to define the system. From the stability analysis within the lubrication approximation, it is evident that the stability condition obtained (Eq. 12) is independent of the presence of particles. The same holds in the non-lubrication analysis and hence the particle conservation equation is excluded.

\subsection{Stability analysis:}

Similar to the lubrication analysis, we perform a stability analysis of the governing equations to obtain the non-uniformity length scales in the shorter range. Henceforth, the over-bars for the dimensionless variables are eliminated for simplicity. The governing equations derived in the previous section are linearized about the base solutions for $u, v, p, h$, and $\Gamma$ using normal modes (in the horizontal direction):

$$
\begin{aligned}
& u=u_{b}+\hat{u}(z) \exp \left(\omega_{c} t+i k_{1} x\right) \\
& v=v_{b}+\hat{v}(z) \exp \left(\omega_{c} t+i k_{1} x\right)
\end{aligned}
$$




$$
\begin{aligned}
& p=p_{b}+\hat{p}(z) \exp \left(\omega_{c} t+i k_{1} x\right) \\
& H=H_{b}+\hat{H} \exp \left(\omega_{c} t+i k_{1} x\right) \\
& \Gamma=\Gamma_{b}+\hat{\Gamma} \exp \left(\omega_{c} t+i k_{1} x\right)
\end{aligned}
$$

where $k_{1}$ is the wavenumber in the $x$ direction. As a consequence of the 2-D form of the Navier-Stokes equations, the amplitudes of the perturbations for $u, v$, and $p$ are functions of $z$ (vertical direction). The functional form of these amplitudes is obtained from the boundary conditions for the variables. The base solutions are the same as in Equation 10, and additionally, $u_{b}=v_{b}=p_{b}=0$. Substituting for $u, v$, and $p$ from Equation 20 into the N-S equations (17 and 18) and the continuity equation, we obtain a set of differential equations for the perturbation amplitudes, $\hat{u}, \hat{v}$, and $\hat{p}$ :

$$
\begin{aligned}
& \hat{u}\left(\omega_{c}+A \varepsilon^{2} k_{1}\right)-A \frac{d^{2} \hat{u}}{d z^{2}}+\left(i k_{1} A\right) \hat{p}=0 \\
& \hat{v}\left(\omega_{c}+A \varepsilon^{2} k_{1}^{2}\right)-A \frac{d^{2} \hat{v}}{d z^{2}}+\left(\frac{A}{\varepsilon^{2}}\right) \frac{d \hat{p}}{d z}=0 \\
& \hat{u}\left(i k_{1}\right)+\frac{d \hat{v}}{d z}=0
\end{aligned}
$$

Adding $\mathrm{ik}_{1} *(21)$ to the $\mathrm{z}$ derivative of (22), and using (23), we obtain the functional form for $\hat{p}$ as

$$
\hat{\mathrm{p}}=\mathrm{C}_{1} \exp \left(\mathrm{k}_{1} \varepsilon \mathrm{z}\right)+\mathrm{C}_{2} \exp \left(-\mathrm{k}_{1} \varepsilon \mathrm{z}\right)
$$

Taking $\hat{p}$ to be zero at $\mathrm{z}=0$, and expanding the exponentials in the limit of $\mathrm{k}_{1} \varepsilon \mathrm{z}<<1$, we obtain

$$
\begin{aligned}
& \left.\hat{\mathrm{p}}\right|_{\mathrm{H}}=2 \mathrm{C}_{1} \mathrm{k}_{1} \varepsilon \mathrm{H}_{\mathrm{b}} \text { and }\left.\frac{\partial \hat{\mathrm{p}}}{\partial \mathrm{z}}\right|_{\mathrm{H}}=2 \mathrm{C}_{1} \mathrm{k}_{1} \varepsilon \text { giving us a relation: } \\
& \left.\frac{d \hat{p}}{d z}\right|_{H}=\frac{\left.\hat{p}\right|_{H}}{H_{b}}
\end{aligned}
$$

where $\left.\frac{d \hat{p}}{d z}\right|_{H}$ and $\left.\hat{\mathrm{p}}\right|_{\mathrm{H}}$ are evaluations at the air-water interface i.e. $z=H$.

Applying the equations (21)-(23) at the air-water interface $(z=H)$, we get

$$
\left.\hat{u}\right|_{H}\left(\omega_{c}+A \varepsilon^{2} k_{1}\right)-\left.A \frac{d^{2} \hat{u}}{d z^{2}}\right|_{H}+\left.\left(i k_{1} A\right) \hat{p}\right|_{H}=0
$$




$$
\begin{aligned}
& \left.\hat{v}\right|_{H}\left(\omega_{c}+A \varepsilon^{2} k_{1}^{2}\right)-\left.A \frac{d^{2} \hat{v}}{d z^{2}}\right|_{H}+\left.\left(\frac{A}{\varepsilon^{2}}\right) \frac{d \hat{p}}{d z}\right|_{H}=0 \\
& \left.\hat{u}\right|_{H}\left(i k_{1}\right)+\left.\frac{d \hat{v}}{d z}\right|_{H}=0
\end{aligned}
$$

Further, substituting for $u, v$, and $p$ from Equation 20 into the kinematic boundary condition (Equation 5), normal stress balance (Equation 3), tangential stress balance, and surfactant conservation equation (Equation 19), we obtain a set of algebraic equations relating the perturbation amplitudes:

$$
\begin{aligned}
& \left.\hat{\mathrm{v}}\right|_{\mathrm{H}}+\omega_{\mathrm{c}} \hat{\mathrm{H}}=0 \\
& \left.\hat{\mathrm{p}}\right|_{\mathrm{H}}-\alpha_{1} \mathrm{k}_{1}{ }^{2} \hat{\mathrm{H}}=0 \\
& \left.\frac{d \hat{u}}{d z}\right|_{H}+i k_{1} \hat{\Gamma}=0
\end{aligned}
$$

$\left.\mathrm{ik}_{1} \Gamma_{\mathrm{b}} \hat{\mathrm{u}}\right|_{\mathrm{H}}+\hat{\Gamma}\left(\omega_{\mathrm{c}}+\frac{\mathrm{k}_{1}^{2}}{\mathrm{Pe}_{\mathrm{s}}}+\alpha_{2} \mathrm{H}_{\mathrm{b}}\right)+\alpha_{2}\left(\Gamma_{\mathrm{b}}-\Gamma_{\mathrm{S}}\right) \hat{\mathrm{H}}=0$

From the continuity equation (23), at $z=H$, we have

$$
\left.\frac{d^{2} \hat{v}}{d z^{2}}\right|_{H}=-\left.i k_{1} \frac{d \hat{u}}{d z}\right|_{H}
$$

Combining this with equation (31), we obtain

$$
\left.\frac{d^{2} \hat{v}}{d z^{2}}\right|_{H}=-k_{1}^{2} \hat{\Gamma}
$$

Substituting for $\left.\hat{\mathrm{u}}\right|_{\mathrm{H}}$ from (32) and $\left.\hat{\mathrm{p}}\right|_{\mathrm{H}}$ from (30), into equation (26), we obtain a linear algebraic equation relating $\hat{H}$ and $\hat{\Gamma}$ :

$$
\left[\mathrm{A} \alpha_{1} \mathrm{k}_{1}{ }^{3} \Gamma_{\mathrm{b}}+\frac{\alpha_{2}\left(\Gamma_{\mathrm{b}}-\Gamma_{\mathrm{s}}\right)\left(\omega_{\mathrm{c}}+\mathrm{A} \varepsilon^{2} \mathrm{k}_{1}^{2}\right)}{\mathrm{k}_{1} \Gamma_{\mathrm{b}}}\right] \hat{\mathrm{H}}+\left[\left(\omega_{\mathrm{c}}+\frac{\mathrm{k}_{1}{ }^{2}}{\mathrm{Pe}_{\mathrm{s}}}+\alpha_{2} \mathrm{H}_{\mathrm{b}}\right) \frac{\left(\omega_{\mathrm{c}}+\mathrm{A} \varepsilon^{2} \mathrm{k}_{1}{ }^{2}\right)}{\mathrm{k}_{1} \Gamma_{\mathrm{b}}}\right] \hat{\Gamma}=0
$$

Similarly, substituting for $\left.\hat{\mathrm{v}}\right|_{\mathrm{H}}$ from (29), $\left.\frac{d^{2} \hat{v}}{d z^{2}}\right|_{H}$ from (34), and $\left.\frac{d \hat{p}}{d z}\right|_{H}$ from (25) and (30), into equation (26), we obtain a second linear algebraic equation relating $\hat{H}$ and $\hat{\Gamma}$ :$$
\left[\omega_{\mathrm{c}}\left(\omega_{\mathrm{c}}+\mathrm{A} \varepsilon^{2} \mathrm{k}_{1}{ }^{2}\right)+\frac{\mathrm{A} \alpha_{1} \mathrm{k}_{1}{ }^{2}}{\varepsilon^{2} \mathrm{H}_{\mathrm{b}}}\right] \hat{\mathrm{H}}+\left[\mathrm{Ak}_{1}{ }^{2}\right] \hat{\Gamma}=0
$$ 
For the system of linear equations 35 and 36 to have non-trivial solutions, the determinant of the coefficient matrix representing them is set to zero i.e.

$$
\begin{aligned}
& {\left[\mathrm{A} \alpha_{1} \mathrm{k}_{1}^{3} \Gamma_{\mathrm{b}}+\frac{\alpha_{2}\left(\Gamma_{\mathrm{b}}-\Gamma_{\mathrm{s}}\right)\left(\omega_{\mathrm{c}}+\mathrm{A} \varepsilon^{2} \mathrm{k}_{1}^{2}\right)}{\mathrm{k}_{1} \Gamma_{\mathrm{b}}}\right]\left[\mathrm{Ak}_{1}^{2}\right]-} \\
& {\left[\left(\omega_{\mathrm{c}}+\frac{\mathrm{k}_{1}^{2}}{\mathrm{Pe}_{\mathrm{s}}}+\alpha_{2} \mathrm{H}_{\mathrm{b}}\right)\left(\frac{\left(\omega_{\mathrm{c}}+\mathrm{A} \varepsilon^{2} \mathrm{k}_{1}^{2}\right)}{\mathrm{k}_{1} \Gamma_{\mathrm{b}}}\right]\left[\omega_{\mathrm{c}}\left(\omega_{\mathrm{c}}+\mathrm{A} \varepsilon^{2} \mathrm{k}_{1}^{2}\right)+\frac{\mathrm{A} \alpha_{1} \mathrm{k}_{1}^{2}}{\varepsilon^{2} \mathrm{H}_{\mathrm{b}}}\right]=0\right.}
\end{aligned}
$$

which gives us a $4^{\text {th }}$ order polynomial equation for growth rate $\omega_{c}$ in terms of the wavenumber $k_{1}$ and the operating parameters. For the operating parameters used here, the polynomial equation has two real roots and a pair of complex conjugate roots. One of the real root ends up being negative, leaving the sign of the $4^{\text {th }}$ root (the other real root) to determine the stability of the system. The next section provides the results from the stability analysis, including the effect of film thickness on the size of nonuniformities observed.

\subsection{Theoretical predictions}

The set of "free parameters" involved in this analysis are $A, \alpha_{1}, \alpha_{2}$, and $P e_{s}$, the physical meanings of which are already defined. For a given set of "free parameter" values, the real root from Equation 37 for growth rate $\omega_{c}$ can be plotted as a function of wavenumber $k_{1}$ (the stability curve). As can be seen from Equation 37, $\omega_{c}$ is a function of the base solution for film height, $H_{b}$. From Equation 10, it is evident that $H_{b}$ is time dependent and hence the stability behaviour evolves with time during drying. In order to obtain a single wavelength that gives the size of non-uniformity for a given set of operating conditions, the growth rate $\omega_{c}$ needs to be integrated over the drying time. This is exactly the same procedure as outlined in the lubrication theory section. When the time integrated growth rate $\varpi\left(k_{1}\right)$ is plotted against the wavenumber $k_{1}$ we obtain a single stability curve representing the stability behaviour under given operating conditions. Figure 10 shows a typical stability curve The operating conditions such as $E, \mu_{0}, \Gamma_{b}$, etc are the same as used in the lubrication analysis. The wavenumber corresponding to the maximum of $\varpi\left(k_{1}\right)$, given the 
symbol $k_{1}{ }^{*}$, gives the dimensionless non-uniformity length scale value, $\lambda_{N U}$. In the non-lubrication analysis, the horizontal length scale $L_{0}$ is obtained by setting $\varepsilon$, the ratio of horizontal and vertical length scales, to 1 . The dimensional value of the nonuniformity length is given by $L_{N U}=\left(\frac{2 \pi}{k_{1}^{*}}\right) L_{0}$.

As the initial film thickness $\left(H_{0}\right)$ has an important effect on the entire film formation process, it is interesting to see what effect it has on the size of the non-uniformities observed in the presence of surfactant. Figure 11 shows the non-uniformity lengths predicted from the non-lubrication theory plotted against the initial film thickness. When the film thickness is varied from 10 to $100 \mu \mathrm{m}$, the predicted size varies from about 50 to $150 \mu \mathrm{m}$. It is interesting to note that $H_{0}$ has the same effect (increasing $L_{N U}$ with increasing $H_{0}$ ) on the size of non-uniformity as in the case of lubrication theory. This suggests that it is the same mechanism (surfactant desorption from the surface) that drives the instability, irrespective of the size of domain under consideration. The only difference in the non-lubrication analysis is that the predicted size is in the micrometer range, while it is in the millimetre range for lubrication theory. This difference can be explained based on the relative strengths of the capillary forces in the two cases. The main effect of changing from a larger domain (lubrication theory) to a smaller domain (non-lubrication theory) is the increase in the strength of capillary forces relative to other forces (as given in the definition of $\alpha_{1}$ ). The effect of stronger capillary forces is to yield the shorter spacing for the island-like surface structures. Hence the predicted length scales in the non-lubrication theory are on much shorter length scales than predicted from the non-lubrication theory.

As the same mechanism drives the instability in lubrication and non-lubrication theories, the system operating conditions are expected to have similar effect on the size of non-uniformities for both cases. Figure 12 shows the effect of desorption strength, $\alpha_{2}$ on the scaled size of non-uniformity, $\lambda_{N U}$. The size of non-uniformity decreases with increasing $\alpha_{2}$, similar to the lubrication theory analysis, but the magnitude of the effect is higher in the non-lubrication analysis. Based on the 
definition for $\bar{\Gamma}_{S}$, the surfactant concentration in the sub-surface, its value can change only over less than an order of magnitude, and hence its effect on the size of nonuniformity is negligible.

The next section provides some experimental evidence to the predictions from the non-lubrication theory.

\section{Experimental investigation of surface instabilities over length scales comparable to the film thickness}

In this section the non-lubrication theory predictions on the effect of initial film thickness and surfactant concentration are verified experimentally. As the predicted non-uniformities are in the $\mu \mathrm{m}$ range, atomic force microscopy (AFM) is a useful tool to make the observations. The materials used and the details of the experimental method are discussed next.

5.1 Materials. A latex was made of a random copolymer based mainly on the following monomers: 2-ethyl hexyl acrylate (2-EHA) (glass transition temperature, $\mathrm{T}_{\mathrm{g}}$ $\left.=-85{ }^{\circ} \mathrm{C}\right)$, ethyl acrylate $\left(\mathrm{T}_{\mathrm{g}}=-22{ }^{\circ} \mathrm{C}\right)$, and methyl methacrylate $(\mathrm{MMA})\left(\mathrm{T}_{\mathrm{g}}=105\right.$ ${ }^{\circ} \mathrm{C}$ ), such that the latex polymer has a $T_{\mathrm{g}}=-50{ }^{\circ} \mathrm{C}$. The latex particles were synthesized by a semi-continuous emulsion polymerization process. The latex polymerization avoided the use of sodium in all components except for the surfactant. To avoid the introduction of sodium, the initiator was chosen to be ammonium persulfate, the buffer was ammonium carbonate, and ascorbic acid was used as a monomer chaser. As sodium was only present in the surfactant phase, time-of-flight secondary ion mass spectrometry (ToF-SIMS) analysis is able to map the distribution of surfactants without ambiguity. The average particle diameter was $226 \mathrm{~nm}$ (measured with quasi-elastic light scattering $\left(\mathrm{Nicomp}^{\mathrm{TM}}, 380 \mathrm{ZLS}\right)$ ) and the solids 
content was 50 wt.\%. In a control experiment, the latex was dialyzed to remove the surfactants and water-soluble polymer in the serum and then examined with ToFSIMS to confirm that $\mathrm{Na}$ species were not found within the polymer. To test the prediction of the non-lubrication model predictions, a blend of surfactants was added to the as-received latex at known concentrations. The blend consists of a 1:1 ratio of ammonium laureth sulfate (ALS) and sodium dodecylbenzene sulfonate (SDBS). Structures are shown in Scheme 1. The as-received latex contained 2 wt.\% surfactant in total, consisting mainly of ALS and SDBS.

5.2 Instrumentation. All AFM measurements reported here were performed using a system (NTEGRA, NT-MDT, Moscow, Russia) with intermittent contact with a silicon cantilever (AC160TN, Olympus). The nominal resonant frequency $f_{o}$ of the cantilever is about $300 \mathrm{kHz}$ and its spring constant $k$ is about $42 \mathrm{~N} / \mathrm{m}$. The optimum method for obtaining images of this type of soft latex surface has been reported previously [12].

A time-of-flight mass spectrometer (TOF-SIMS5-100, ION-TOF GmbH, Heisenbergstr) employing a $\mathrm{Bi}^{3+}$ beam was used to extract elemental and molecular spectra as a function of lateral position and thereby to produce maps of elements and molecules on the sample surface.

5.3 Film Preparation. Films were cast onto PET substrates $(30 \mathrm{~cm} \times 20 \mathrm{~cm})$ using a hand-held bar coater with a defined wet thickness ranging from $30 \mu \mathrm{m}$ to $200 \mu \mathrm{m}$. The films were then dried in ambient conditions. The evaporation rate, $E$, under laboratory conditions is on the order of $1 \times 10^{-8} \mathrm{~m} \mathrm{~s}^{-1}$, which is of the same order of 
magnitude used in our earlier analysis herein. It takes more than $2 \mathrm{hr}$ for a $200 \mu \mathrm{m}$ wet film to dry under these conditions.

5.4 Experimental Results. When the surfactant blend was added to the latex, islandlike features were always found via AFM analysis of the air surface of films dried at room temperature. Figure 13(a) provides an example of a latex film with 2 wt.\% added surfactant (bringing the total concentration to 4 wt. $\%$ in the dry film). In the corresponding ToF-SIMS mapping of the same surface, shown in Figure 13(b), the "islands" give a strong signal with a mass of $63 \mathrm{amu}$, which is attributed to the $\mathrm{Na}_{2} \mathrm{OH}^{+}$ion, which must arise from the surfactant as the only $\mathrm{Na}$ source. (Note that preliminary SIMS analysis of the dialysed latex found no $\mathrm{Na}_{2} \mathrm{OH}^{+}$signal, whereas it was found as a signature in the SIMS analysis of neat SDBS.)

This interpretation is supported by complementary experiments: Rinsing the surface with water causes the islands to disappear from the surface, thus confirming the island structures are water-soluble, as expected for surfactant. Furthermore, as a small molecule, the surfactant is expected to be more liquid-like and less energy dissipative in comparison to the highly viscous polymer. Our previous work [12] has reported this difference in energy dissipation, leading to surfactant features appearing lighter in the phase images, as is observed here. We conclude that the islands are a surfactantrich region.

It can be seen from AFM height images (not shown here) that surfactant islands are lower than the surrounding surface. When the latex films were rinsed with water, the surfactant islands disappeared and left permanent indentations or hollows on the film 
surface, as already reported [12]. Such surfactant aggregation on the surface is a consequence of surfactant convection and spreading on the surface.

Further experiments were conducted to determine the key factors that determine the average size and spacings of the surfactant islands. The surfactant blend (1:1 ratio of ALS and SDBS) was added at a known concentration to the dialyzed latex and the film thickness was varied. An example is shown in Figure 14 in which 2 wt.\% surfactant was added to the dialyzed latex, and films were cast onto PET substrates with wet thicknesses (initial film thickness $H_{0}$ ) varying from 30 to $120 \mu \mathrm{m}$. The images show that the size of the surfactant islands and the spacing between the islands, $\boldsymbol{L}_{N \boldsymbol{U}}$, increase with increasing $H_{0}$. Figure 15 quantitatively shows this dependence after analysis of the images in Figure 14. The error bars on the data points represent the standard deviation obtained from multiple measurements. The observed sizes range from a few tens of $\mu \mathrm{m}$ up to a value on the order of $100 \mu \mathrm{m}$, as predicted in the non-lubrication theory (Figure 11). This broad agreement between experiment and theoretical prediction is satisfactory considering the limitations in the predictions resulting from the undetermined value of $\alpha_{2}$, i.e. $\alpha_{2}$, the desorption strength, is not experimentally determined and the value used in the theory is an estimated one.

Experiments were also conducted to see the effect of excess surfactant concentration. Additional surfactant (a 1:1 blend of ALS and SDBS) was added to the as-prepared latex (having 2 wt.\% surfactant) in various concentrations. AFM images indicate that the Spacing between the surfactant islands increases with the surfactant concentration, 
as shown by the representative images in Figure 16. While non-lubrication theory predicts $\bar{\Gamma}_{S}$ to have little effect on the size of non-uniformity (Section 4.3), AFM images indicate that island separation is influenced by surfactant concentration. The discrepancy could be due to the undetermined value of $\alpha_{2}$ or it more likely implies that the bulk surfactant concentration, $\bar{\Gamma}_{S}$, and the surface concentration are not related as simply as has been assumed and at higher surfactant concentrations a more complex isotherm is evident with a bilayer or similar structure forming.

\section{Summary of the findings:}

The present work identifies two instabilities arising during the drying of latex in the presence of surfactant. The two instabilities occur over vastly different length scales and have been observed experimentally. Figure 17 sketches the overlap of the two instabilities, with the long length scale undulations overlaid with the short length scale variation. The theoretical work identified an instability based on desorption of surfactant from the surface to the bulk. The instability was investigated both within the lubrication approximation and also without this assumption. The effect of various operating (and drying) parameters on the wavelengths associated with these two analyses is studied, giving us an understanding of the ways to gain control over the instabilities.

Although the experimental evidence is for two instabilities, the theoretical work has identified a single mechanism and hence a single instability. The lubrication approximation merely indicates the presence of an instability, and it is properly captured by the 2-D theory. In most applications, the instabilities are unwanted, but there are applications, e.g. decorative coatings on ceramics, where a specific surface texture will be desirable. This analysis will allow prediction of the conditions where a desired surface topography will develop.

\section{Conclusions}


With an aim to obtain the length-scales of film thickness non-uniformities in latex films containing surfactant, a linear stability analysis was performed. For fixed drying conditions, the length-scales are given by the wavelengths corresponding to the fastest growing mode of a disturbance. The study, involving both lubrication theory and nonlubrication analysis, predicts the existence of film thickness non-uniformities over micrometer length scales. Experimental observations through direct visualization and AFM indicate the existence of two instability length scales. The study showed that latex dispersions with higher initial particle volume fractions resulted in films with smaller sizes of surface non-uniformities. The length-scales were shown to be independent of the drying rate and diffusive strength of surfactant at the surface. Stronger surfactant desorption into the bulk serum and thinner films lead to smaller non-uniformity length scales. Increasing the surfactant amount in the latex dispersion leads to larger non-uniformity length scales. This study helps to predict the drying (and operating) conditions under which surface non-uniformities can be minimized.

\section{Acknowledgements}

This work is supported by the European Commission through the Framework 6 projects NAPOLEON (Contract No. IP 011844-2) and NsHAPe (Contract No. 505442). The authors would like to thank Colm Caulfield (University of Cambridge) for useful discussions on the stability analysis and Prof. Peter Lovell, Michael Rabjohns and Andrew Foster (University of Manchester) for useful discussions on surfactant in latex. We thank Steven J. Hinder (University of Surrey) for assistance with the ToF-SIMS analysis and Maria Paulis and Maria J. Barandiaran (University of the Basque Country) for providing the BA/MMA latex. 


\section{List of Figures}

Figure 1 Stability curves at different stages of drying for parameters listed in set 1 (Table 1) and using $\Gamma_{b}=1 ; \Gamma_{S}=0.5$

Figure 2 Plot of time integrated growth rate $\varpi(k)$ as a function of wavenumber $k$ for parameters listed in set 1 (Table 1 ) and using $\Gamma_{b}=1 ; \Gamma_{S}=0.5$.

Figure 3 Plot of time integrated growth rate $\varpi(k)$ as a function of wavenumber $k$ for parameters listed in set 2 (Table 1) and using $\Gamma_{b}=1 ; \Gamma_{S}=0.5$.

Figure 4 Dimensionless non-uniformity length scale as a function of initial particle volume fraction with parameters listed in set 1 (Table 1) and using $\Gamma_{b}=1 ; \Gamma_{S}=0.5$.

Figure 5 Stability curves at $t=0$ for various initial particle volume fractions with parameters listed in set 2 (Table 1 ) and using $\Gamma_{b}=1 ; \Gamma_{S}=0.5$.

Figure 6 Dimensionless non-uniformity length scale, $\lambda_{N U}$, as a function of $\alpha_{2}$ with parameters listed in set 2 (Table 1 ) and using $\Gamma_{b}=1 ; \Gamma_{S}=0.5$.

Figure 7 Dimensional non-uniformity length scale as a function of initial film thickness, using $\Gamma_{b}=1 ; \Gamma_{S}=0.5 ; H_{0}=50 \mu \mathrm{m} ; E=3.5^{*} 10^{-8} \mathrm{~m} / \mathrm{s}$; $\mu_{0}=10^{-3} \mathrm{~kg} / \mathrm{m} . \mathrm{s} ; S=4 * 10^{-3} ; \phi_{0}=0.4$.

Figure 8 Dimensionless non-uniformity length scale as a function of $\bar{\Gamma}_{S}$ using $\Gamma_{b}=1 ; H_{0}=50 \mu \mathrm{m} ; E=3.5^{*} 10^{-8} \mathrm{~m} / \mathrm{s} ; \mu_{0}=10^{-3} \mathrm{~kg} / \mathrm{m} . \mathrm{s} ; S=4 * 10^{-3} ; \phi_{0}=0.4$.

Figure 9 Experimentally observed spacing of latex surface striations for films with varying initial film thickness, $H_{0}$

Figure 10 Plot of time integrated growth rate $\varpi\left(k_{1}\right)$ as a function of wavenumber $k_{1}$. using $\Gamma_{b}=1 ; \Gamma_{S}=0.5, H_{0}=100 \mu ; E=3.5 * 10^{-8} \mathrm{~m} / \mathrm{s} ; \mu_{0}=10^{-3} \mathrm{~kg} / \mathrm{m} . \mathrm{s}$; $S=4 * 10^{-3} ; \phi_{0}=0.4$.

Figure 11 Dimensional non-uniformity length scale as a function of initial film thickness, $\Gamma_{b}=1 ; \Gamma_{S}=0.5, H_{0}=100 \mu ; E=3.5 * 10^{-8} \mathrm{~m} / \mathrm{s} ; \mu_{0}=10^{-3} \mathrm{~kg} / \mathrm{m} . \mathrm{s}$; $S=4 * 10^{-3} ; \phi_{0}=0.4$. 
Figure 12 Dimensionless non-uniformity length scale as a function of desorption strength $H_{0}=100 \mu ; E=3.5 * 10^{-8} \mathrm{~m} / \mathrm{s} ; \mu_{0}=10^{-3} \mathrm{~kg} / \mathrm{m} . \mathrm{s} ; S=4 * 10^{-3} ; \phi_{0}=0.4$.

Figure 13 (a) $20 \mu \mathrm{m} \times 20 \mu \mathrm{m}$ AFM images (height on left, phase on right) of the asreceived latex with 2 wt.\% added surfactant. (b) The corresponding $17 \mu \mathrm{m}$ x17 $\mu \mathrm{m}$ ToF-SIMS maps of the same latex film, showing (left to right) $\mathrm{Na}+, \mathrm{Na}_{2} \mathrm{OH}+$ and total positive ions. Lighter color in the map indicates higher ion concentrations.

Figure 14 AFM phase images of the air surface of latex films with increasing wet thickness, from left to right: $30 \mu \mathrm{m}, 60 \mu \mathrm{m}, 90 \mu \mathrm{m}$, and $120 \mu \mathrm{m}$. All image sizes are $100 \mu \mathrm{m} \times 100 \mu \mathrm{m}$.

Figure 15 The dependence of surfactant "island" lateral spacing, $L_{N U}$, on wet latex film thickness, $H_{o}$, from analysis of the images in Figure 14. The solid line is a guide to the eye.

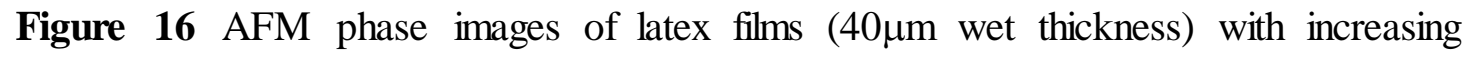
excess surfactant concentrations: (a) as-received; (b) 2 wt.\%; and (c) 4 wt.\%. Image sizes are $20 \mu \mathrm{m} \times 20 \mu \mathrm{m}$.

Figure 17 Sketch showing the overlap of the two instabilities.

Scheme 1. Chemical structure of ALS (left) and SDBS (right).

Table 1 Two different sets of "free parameter" values with constant operating conditions using $H_{0}=50 \mu \mathrm{m} ; E=3.5 \times 10^{-8} \mathrm{~m} / \mathrm{s} ; \mu_{0}=10^{-3} \mathrm{~kg} / \mathrm{m} . \mathrm{s} ; S=4 \times 10^{-2}$ 


\section{References}

1. Winnik M. A., Latex film formation. Current Opinion in Colloid and Interface Science, 1997. 2(2): p. 192.

2. Vanderhoff J.W., Mechanism of film formation of latexes. British Polymer Journal, 1970. 2: p. 161.

3. Rosen M.J., Surfactants and Interfacial Phenomena. 1978, Wiley-Interscience. New York.

4. Kientz E. and Holl Y., Distribution of surfactants in latex films. Colloids and Surfaces A: Physicochemical and Engineering Aspects, 1993. 78: p. 255.

5. Gundabala V. R., Zimmerman W. B., and Routh A. F., A model for surfactant distribution in latex coating. Langmuir, 2004. 20: p. 8721.

6. Keddie J. L., Film formation of latex. Materials Science and Engineering R: Reports, 1997. 21: p. 101.

7. Routh A. F. and Russel W. B., Horizontal drying fronts during solvent evaporation from latex films. AICHE Journal, 1998. 44(9): p. 2088.

8. Belaroui F., Hirn M. P., Grohens Y., Marie P., and Holl Y., Distribution of water-soluble and surface-active low-molecular-weight species in acrylic latex films. Journal of Colloid and Interface Science, 2003. 261: p. 336.

9. Butt H.J., Kuropka R., and Christensen B., Latex film formation studied with the atomic force microscope: Influence of aging and annealing. Colloid and Polymer Science, 1994. 272: p. 1218.

10. Zhao Y. and M.W. Urban, Mobility of SDOSS powered by ionic interactions in Sty/n-BA/MAA core-shell latex films. 21. A spectroscopic study. Langmuir, 2000. 16: p. 9439.

11. Aramendia E., Barandiaran M. J., Grade J., Blease T., and Asua J. M., Improving water sensitivity in acrylic films using surfmers. Langmuir, 2005. 21: p. 1428.

12. Lei C.H., Ouzineb K., Dupont O., and Keddie J.L., Probing particle structure in waterborne pressure sensitive adhesives with atomic force microscopy. Journal of Colloid and Interface Science, 2007. 307: p. 56.

13. Gundabala V. R. and Routh A. F., Thinning of drying latex films due to surfactant. Journal of Colloid and Interface Science, 2006. 303(1): p. 306. 
14. Troian S.M., Wu X.L., and Safran S.A., Fingering instability in thin wetting films. Physical Review Letters, 1989. 62(13): p. 1496.

15. Troian S.M., Herbolzheimer E., and Safran S.A., Model for the fingering instability of spreading surfactant drops. Physical Review Letters, 1990. 65(3): p. 333.

16. Matar O.K. and S.M. Troian, Linear stability analysis of an insoluble surfactant monolayer spreading on a thin liquid film. Physics of Fluids, 1997. 9: p. 3645 .

17. Matar O.K. and Troian S.M., Growth of non-modal transient structures during the spreading of surfactant coated films. Physics of Fluids, 1998. 10(5): p. 1234.

18. Matar O.K. and Troian S.M., Spreading of a surfactant monolayer on a thin liquid film: Onset and evolution of digitated structures. Chaos, 1999. 9(1): p. 141.

19. Sharma A. and Ruckenstein E., The role of lipid abnormalities, aqueous and mucus deficiencies in the tear film breakup, and implications for tear substitutes and contact lens tolerance. Journal of Colloid and Interface Science, 1986. 111(1): p. 8.

20. Matar O.K. and Kumar S., Rupture of surfactant-covered thin liquid films on a flexible wall. SIAM Journal of Applied Mathematics, 2004. 64(6): p. 2144.

21. Kumar S. and Matar O.K., Instabiltiy of long-wavelength disturbances on gravity-modulated surfactant-covered thin liquid layers. Journal of Fluid Mechanics, 2002. 466: p. 249.

22. Danov K.D., Paunov V.N., Stoyanov S.D., Alleborn N., Raszillier H., and Durst F., Stability of evaporating two-layered liquid film in the presence of surfactant - II. Linear analysis. Chemical Engineering Science, 1998. 53(15): p. 2823.

23. Warner M.R.E., Craster R.V., and Matar O.K., Surface patterning via evaporation of ultrathin films containing nanoparticles. Journal of Colloid and Interface Science, 2003. 267: p. 92.

24. Warner M.R.E., Craster R.V., and Matar O.K., Fingering phenomena associated with insoluble surfactant spreading on thin liquid films. Journal of Fluid Mechanics, 2004. 510: p. 169. 
25. Gaver D.P. and Grotberg J.B., Droplet spreading on a thin viscous film. Journal of Fluid Mechanics, 1992. 235: p. 399.

26. Krieger I.M. and Dougherty T.J., A Mechanism for Non-Newtonian Flow in Suspensions of Rigid Spheres. Journal of Rheology, 1959. 3(1): p. 137.

27. Otis D.R., Ingenito E.P., Kamm R.D., and Johnson M., Dynamic surface tension of surfactant TA: experiments and theory. Journal of Applied Physiology, 1994. 77(6): p. 2681.

28. Oron A., Davis S.H., and Bankoff S.G., Long-scale evolution of thin liquid films. Reviews of Modern Physics, 1997. 69(3): p. 931.

29. Drazin P.G. and Reid W.H., Hydrodynamic stability. 1981, Cambridge University Press. Cambridge.

30. Jensen O.E. and Grotberg J. B., The spreading of heat or soluble surfactant along a thin liquid film, Physics of Fluids A, 1993 5(1): p.58. 


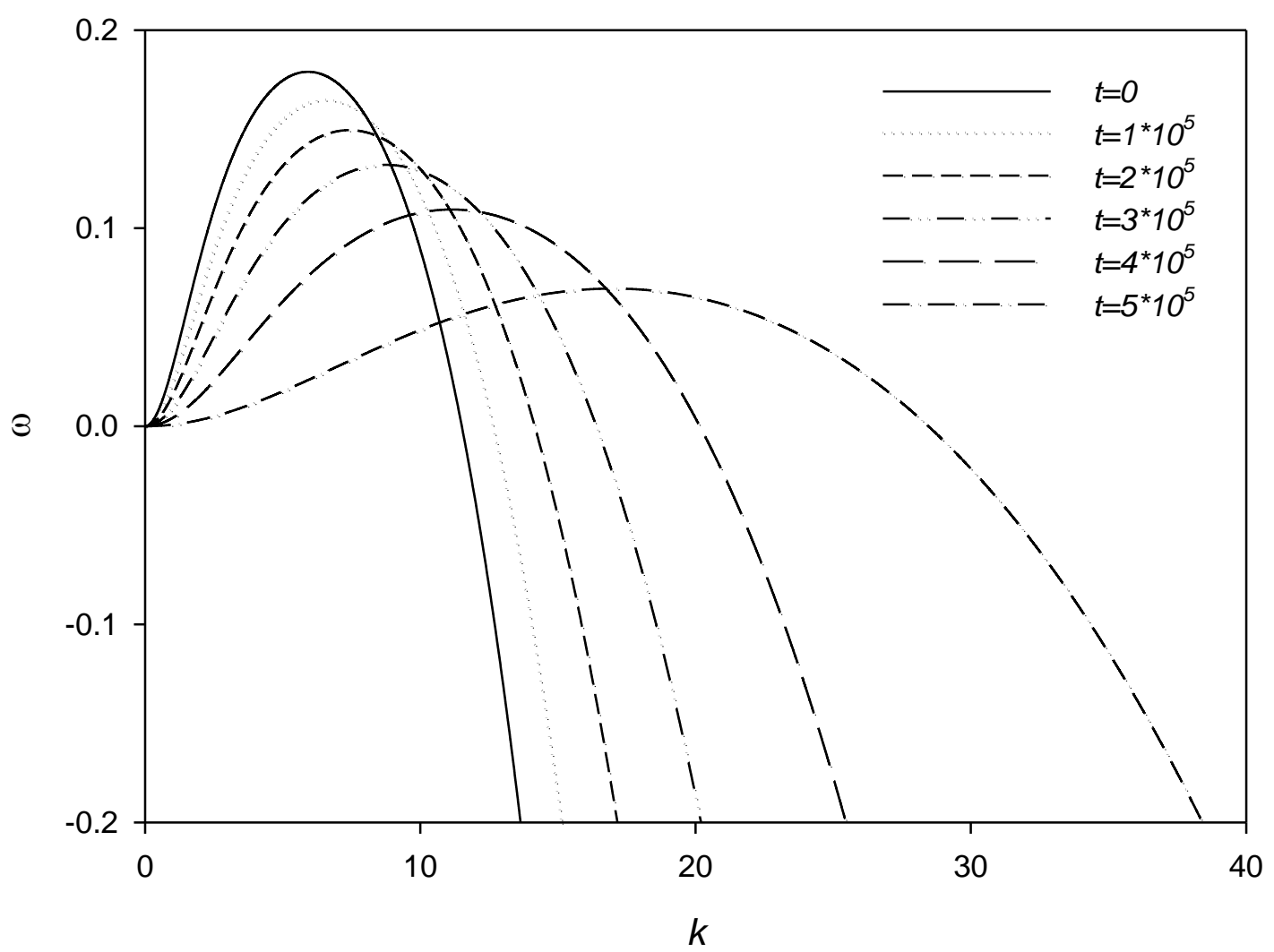

Figure 1 Stability curves at different stages of drying for parameters listed in set 1 (Table 1) and using $\Gamma_{b}=1 ; \Gamma_{S}=0.5$. 


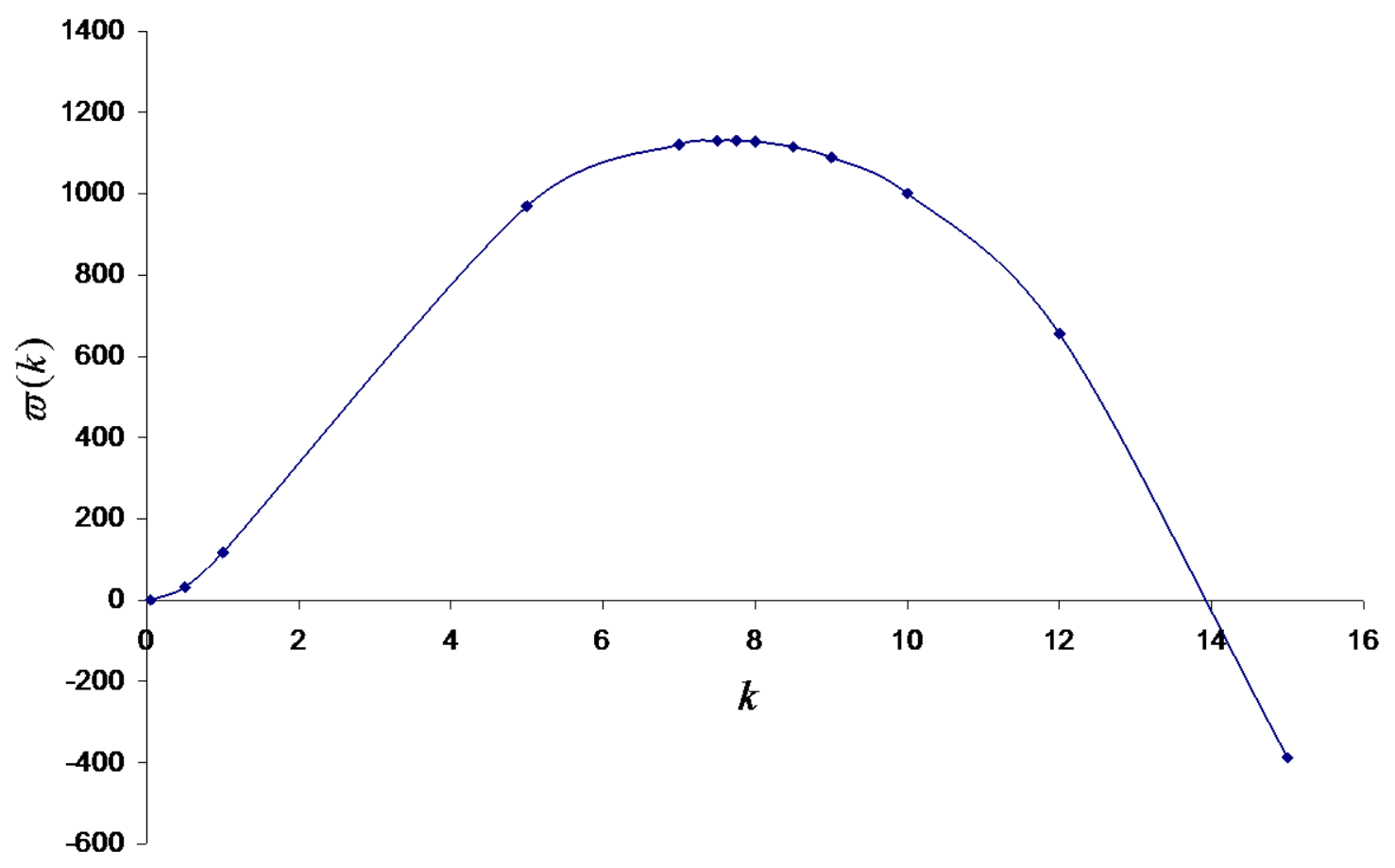

Figure 2 Plot of time integrated growth rate $\varpi(k)$ as a function of wavenumber $k$ for parameters listed in set 1 (Table 1 ) and using $\Gamma_{b}=1 ; \Gamma_{S}=0.5$. 


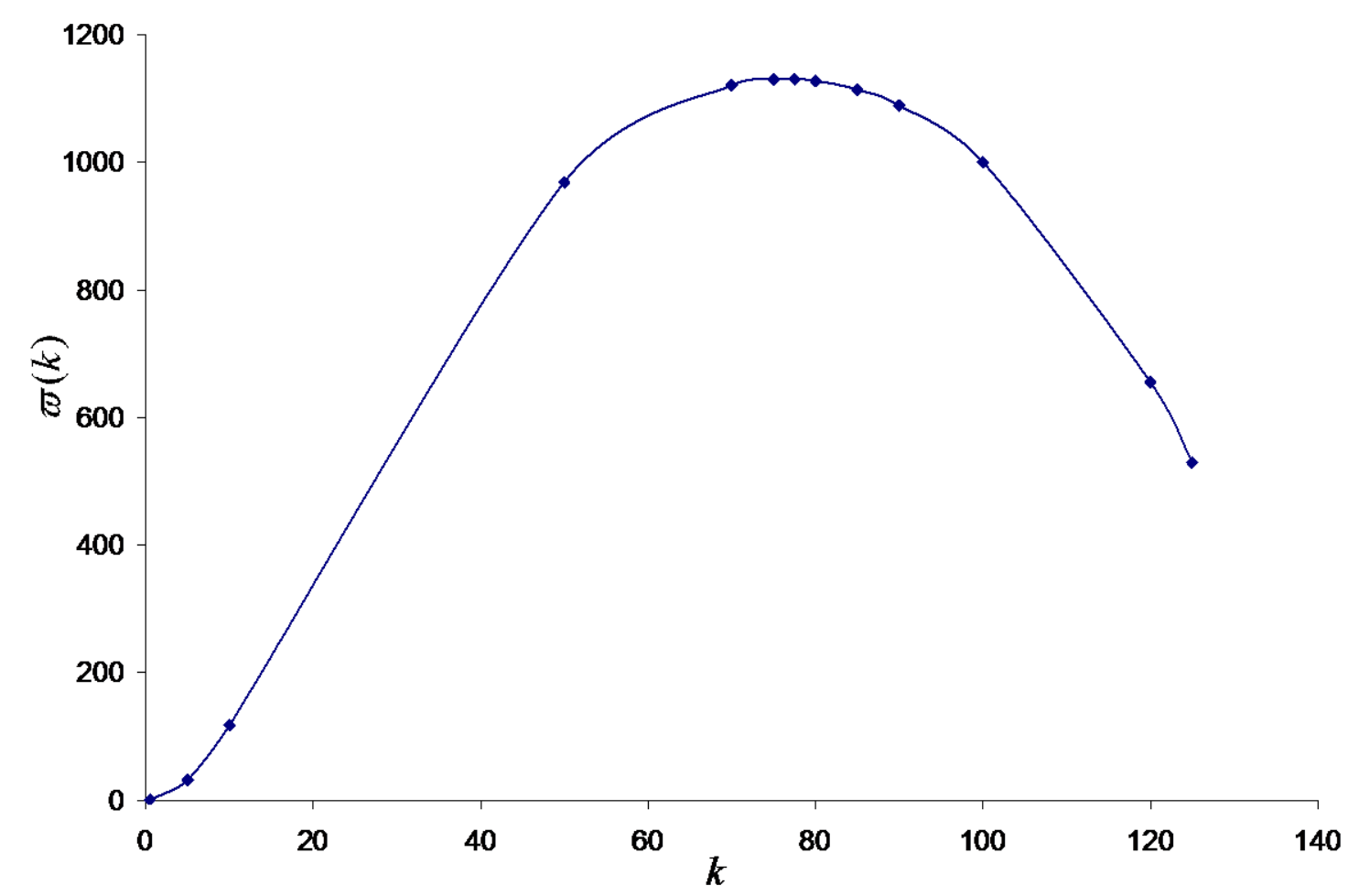

Figure 3 Plot of time integrated growth rate $\varpi(k)$ as a function of wavenumber $k$ for parameters listed in set 2 (Table 1) and using $\Gamma_{b}=1 ; \Gamma_{S}=0.5$. 
Published in JAIChE 54 (2008) 3092-3105

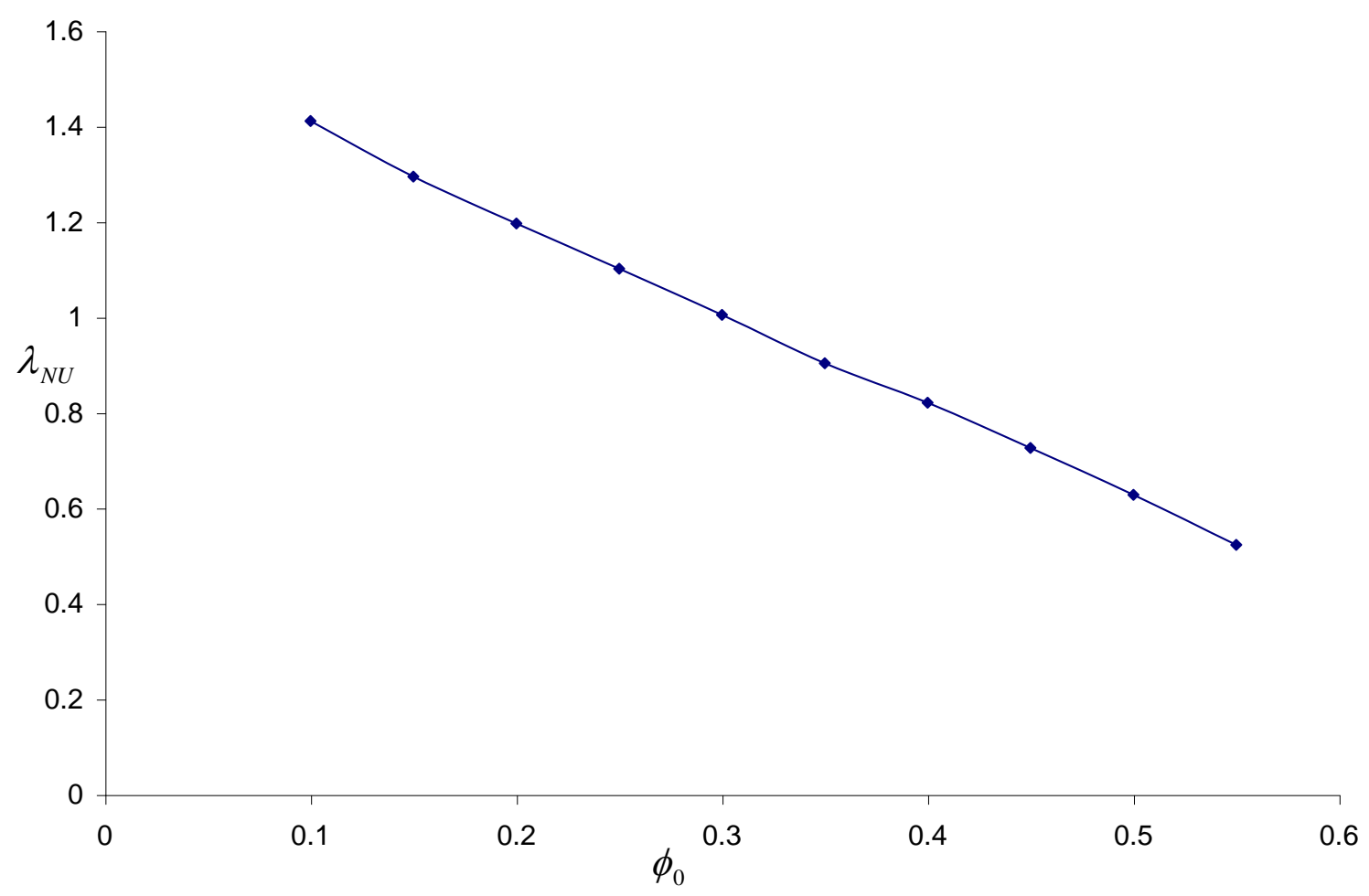

Figure 4 Dimensionless non-uniformity length scale as a function of initial particle volume fraction with parameters listed in set 1 (Table 1) and using $\Gamma_{b}=1 ; \Gamma_{S}=0.5$. 


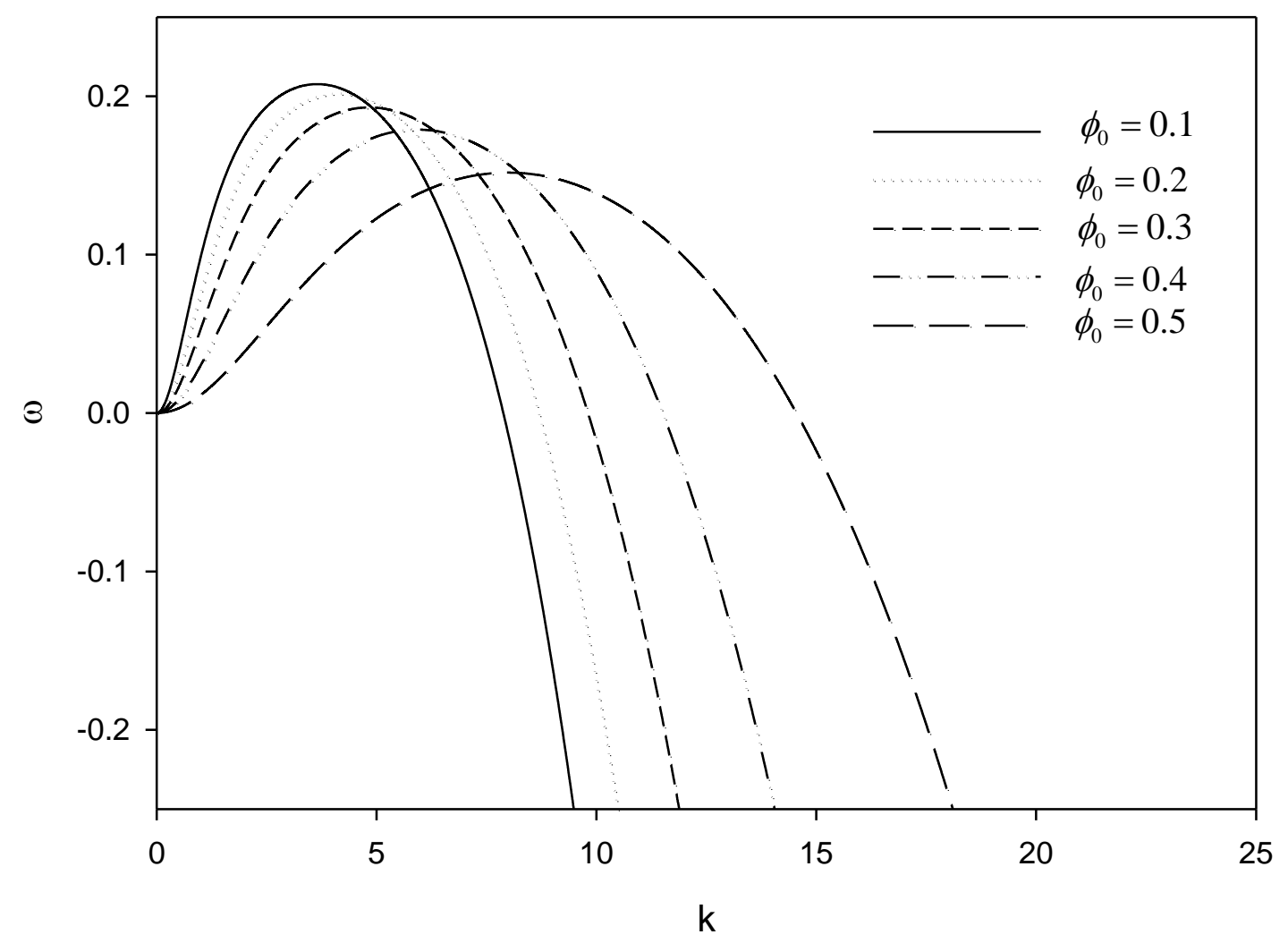

Figure 5 Stability curves at $t=0$ for various initial particle volume fractions with parameters listed in set 2 (Table 1 ) and using $\Gamma_{b}=1 ; \Gamma_{S}=0.5$. 
Published in JAIChE 54 (2008) 3092-3105

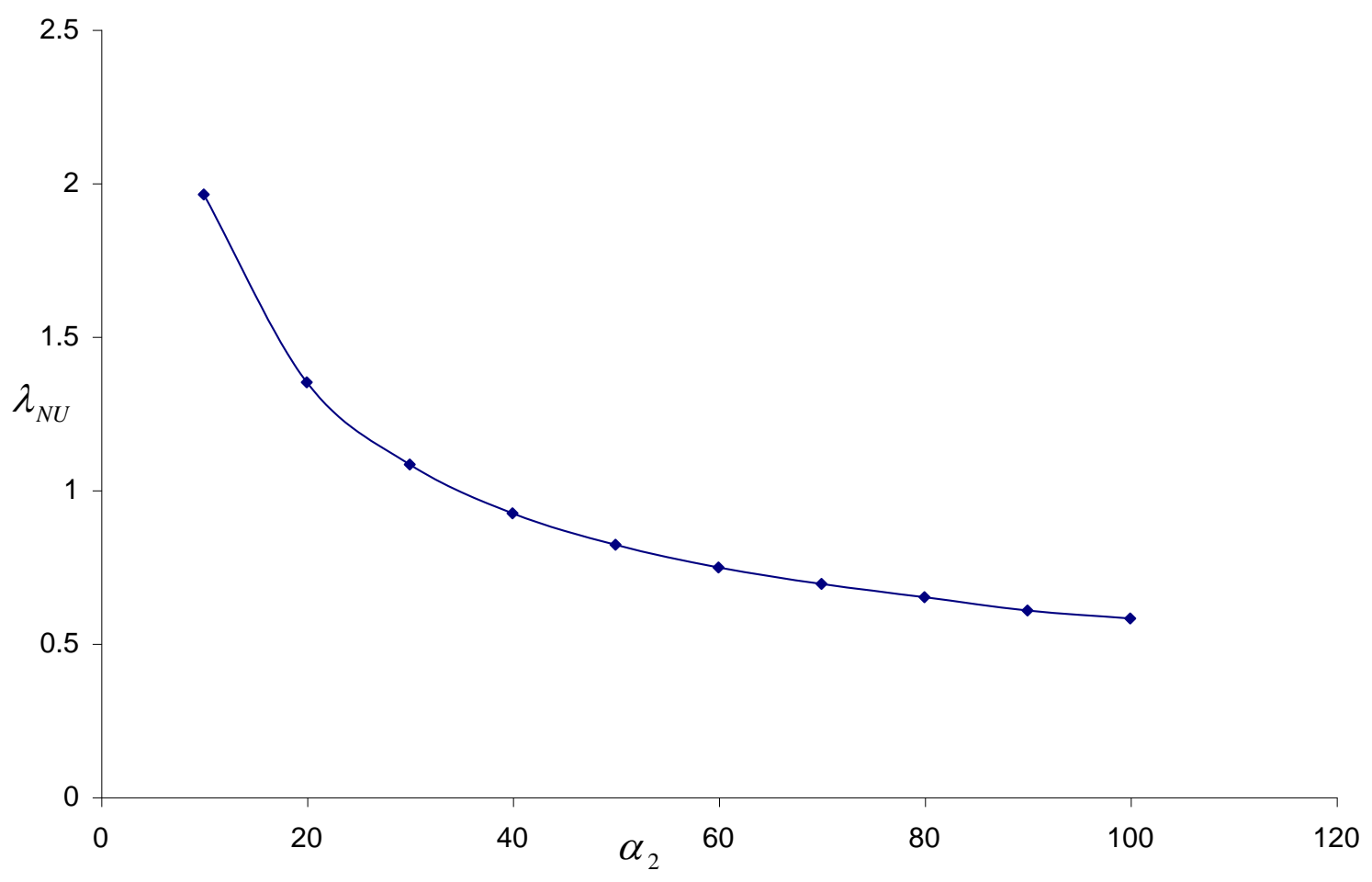

Figure 6 Dimensionless non-uniformity length scale, $\lambda_{N U}$, as a function of $\alpha_{2}$ with parameters listed in set 2 (Table 1 ) and using $\Gamma_{b}=1 ; \Gamma_{S}=0.5$. 


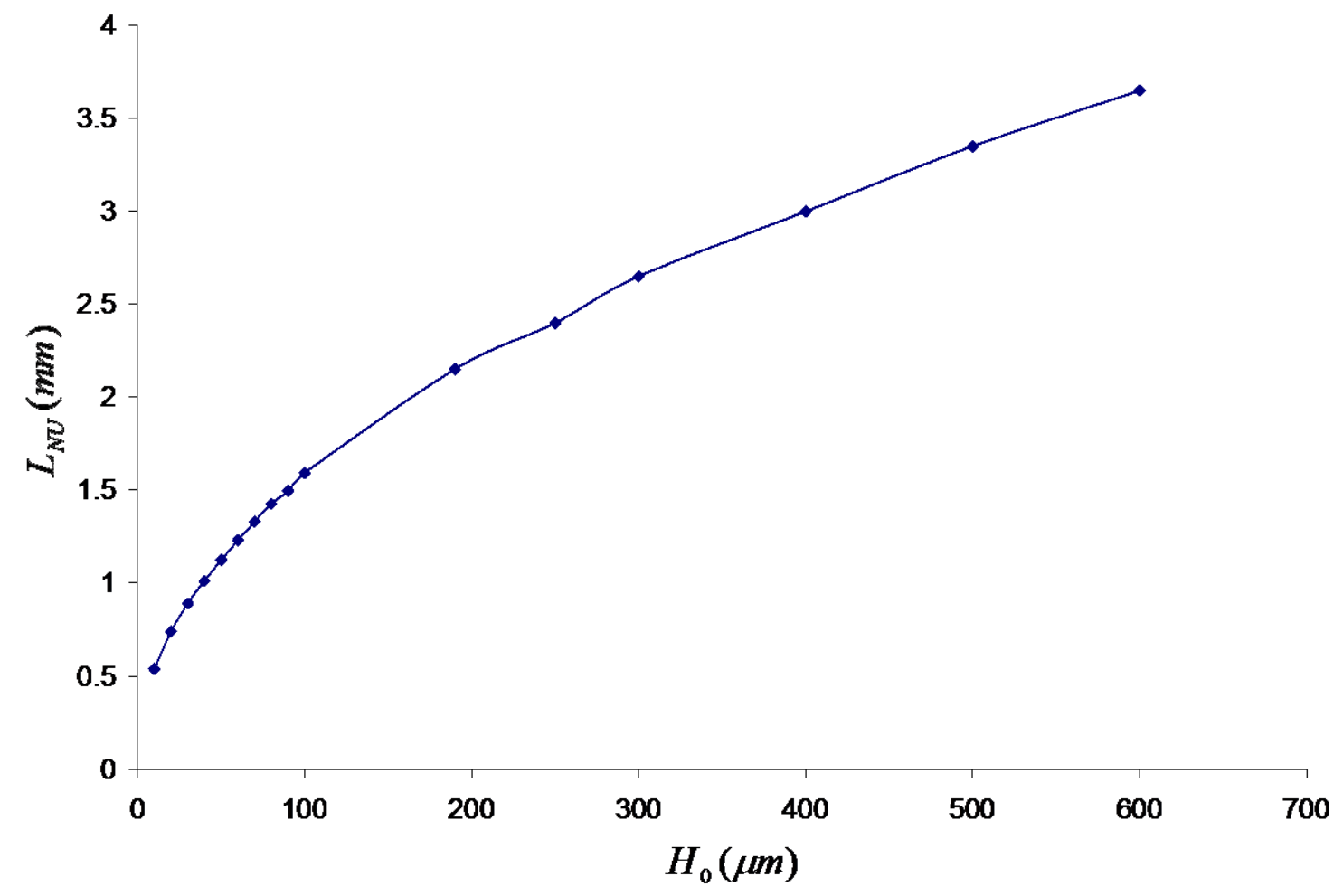

Figure 7 Dimensional non-uniformity length scale as a function of initial film thickness, using $\Gamma_{b}=1 ; \Gamma_{S}=0.5 ; H_{0}=50 \mu \mathrm{m} ; E=3.5 * 10^{-8} \mathrm{~m} / \mathrm{s}$; $\mu_{0}=10^{-3} \mathrm{~kg} / \mathrm{m} . \mathrm{s} ; S=4 * 10^{-3} ; \phi_{0}=0.4$. 
Published in JAIChE 54 (2008) 3092-3105

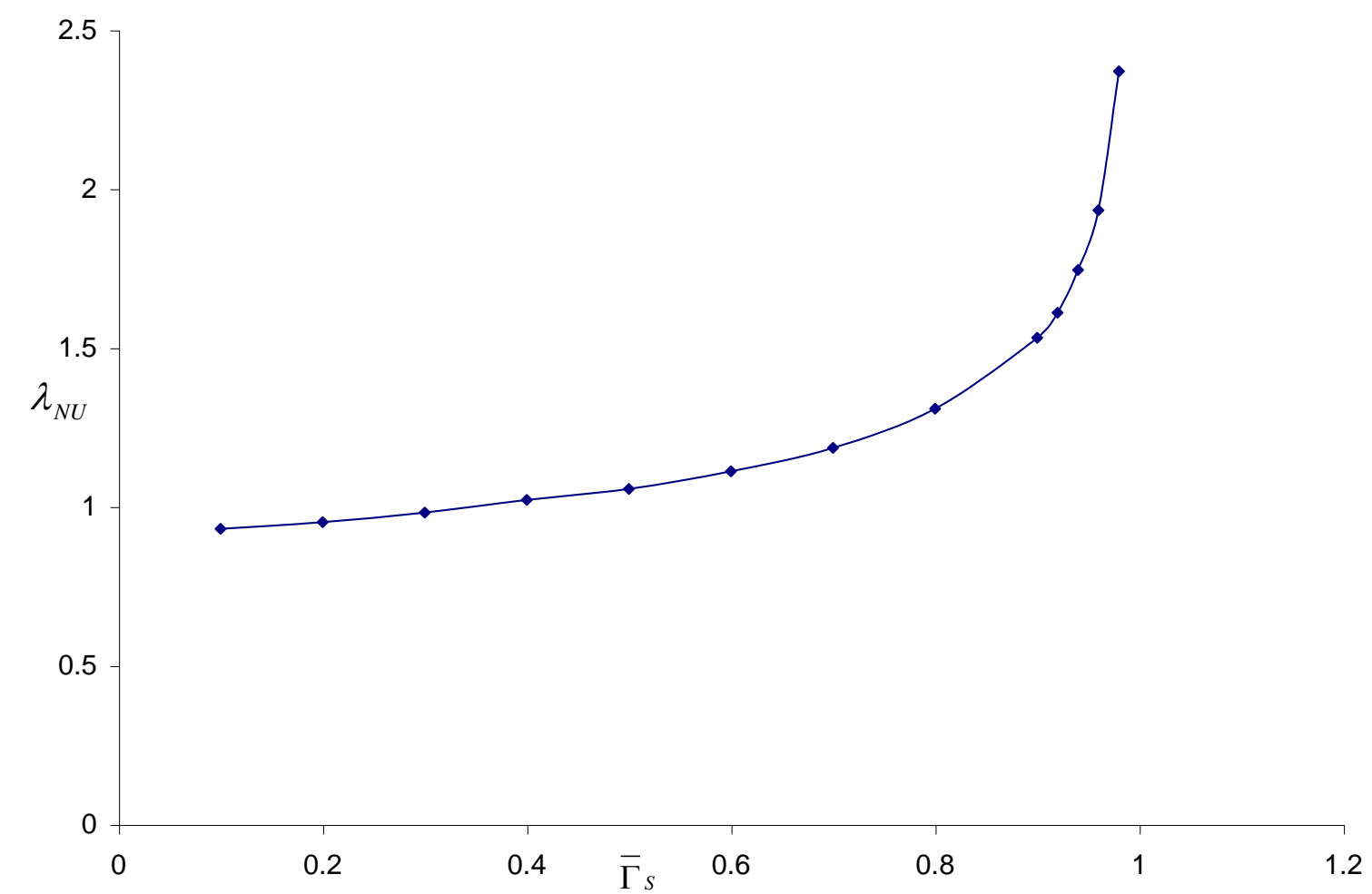

Figure 8 Dimensionless non-uniformity length scale as a function of $\bar{\Gamma}_{s}$ using $\Gamma_{b}=1 ; H_{0}=50 \mu \mathrm{m} ; E=3.5^{*} 10^{-8} \mathrm{~m} / \mathrm{s} ; \mu_{0}=10^{-3} \mathrm{~kg} / \mathrm{m} . \mathrm{s} ; S=4 * 10^{-3} ; \phi_{0}=0.4$. 
Published in JAIChE 54 (2008) 3092-3105

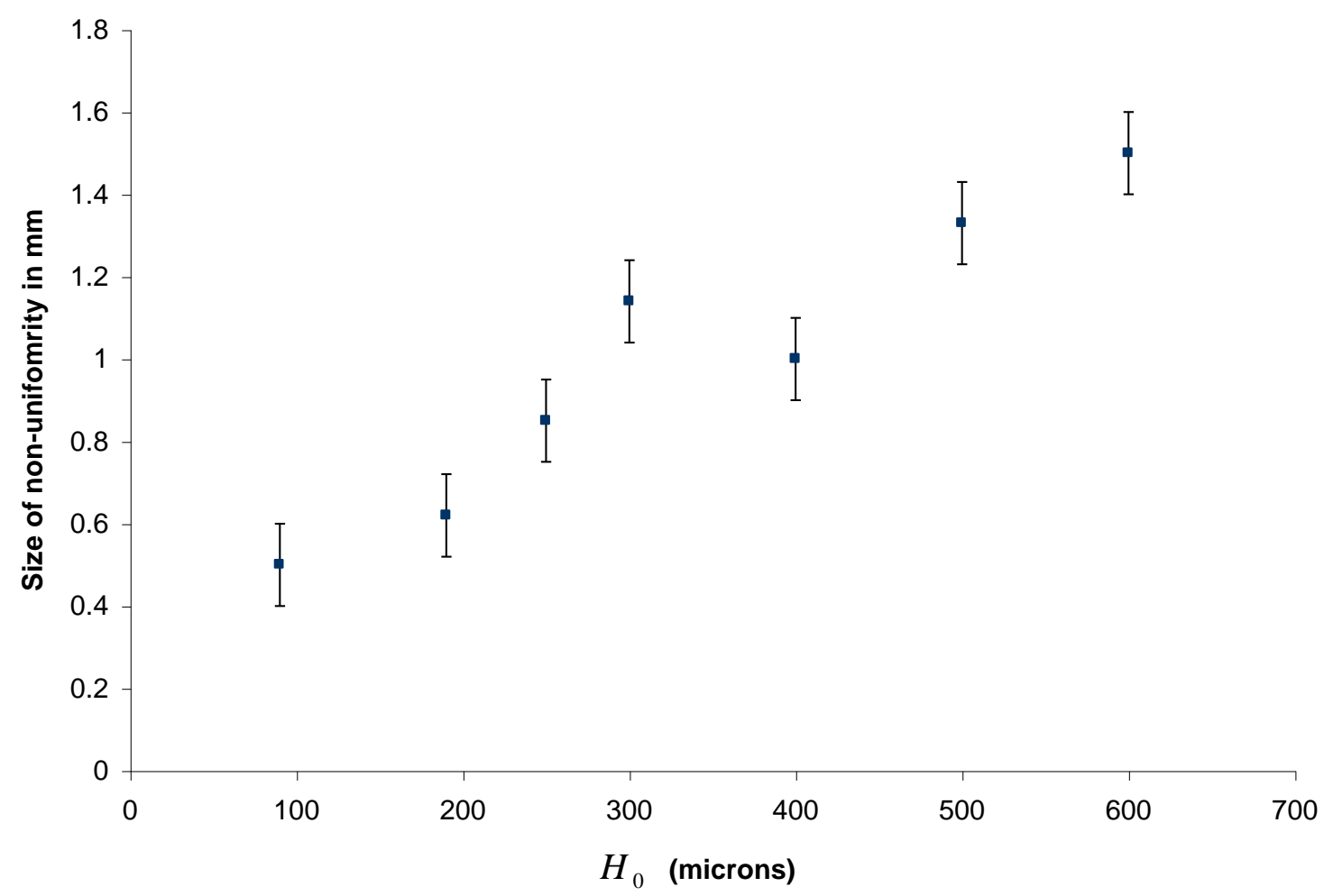

Figure 9 Experimentally observed spacing of latex surface striations for films with varying initial film thickness, $H_{0}$. 


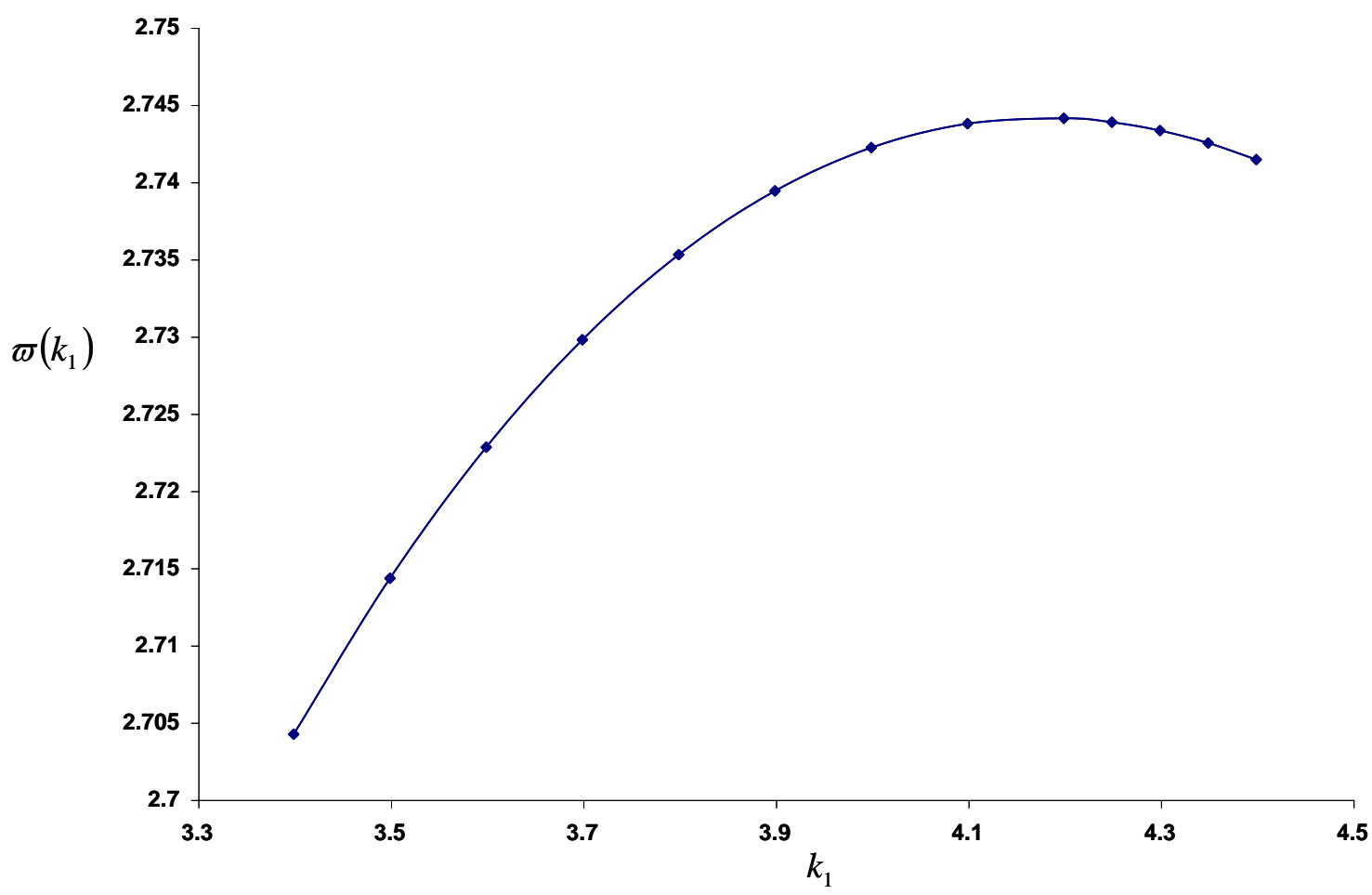

Figure $10 \mathrm{Plot}$ of time integrated growth rate $\varpi\left(k_{1}\right)$ as a function of wavenumber $k_{1}$. using $\Gamma_{b}=1 ; \Gamma_{S}=0.5, H_{0}=100 \mu ; E=3.5 * 10^{-8} \mathrm{~m} / \mathrm{s} ; \mu_{0}=10^{-3} \mathrm{~kg} / \mathrm{m} . \mathrm{s}$; $S=4 * 10^{-3} ; \phi_{0}=0.4$. 


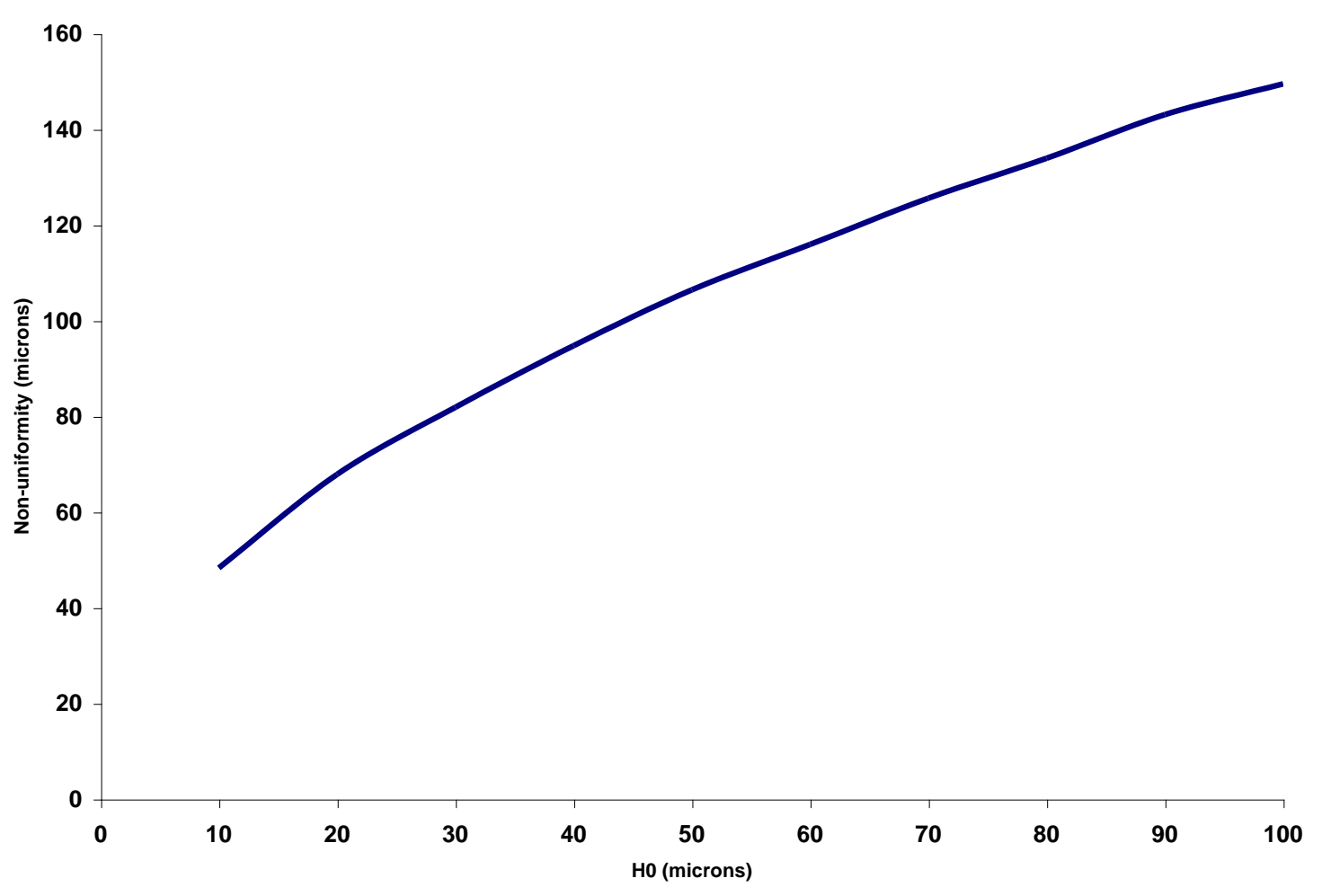

Figure 11 Dimensional non-uniformity length scale as a function of initial film thickness, $\Gamma_{b}=1 ; \Gamma_{S}=0.5, H_{0}=100 \mu ; E=3.5 * 10^{-8} \mathrm{~m} / \mathrm{s} ; \mu_{0}=10^{-3} \mathrm{~kg} / \mathrm{m} . \mathrm{s}$; $S=4 * 10^{-3} ; \phi_{0}=0.4$. 


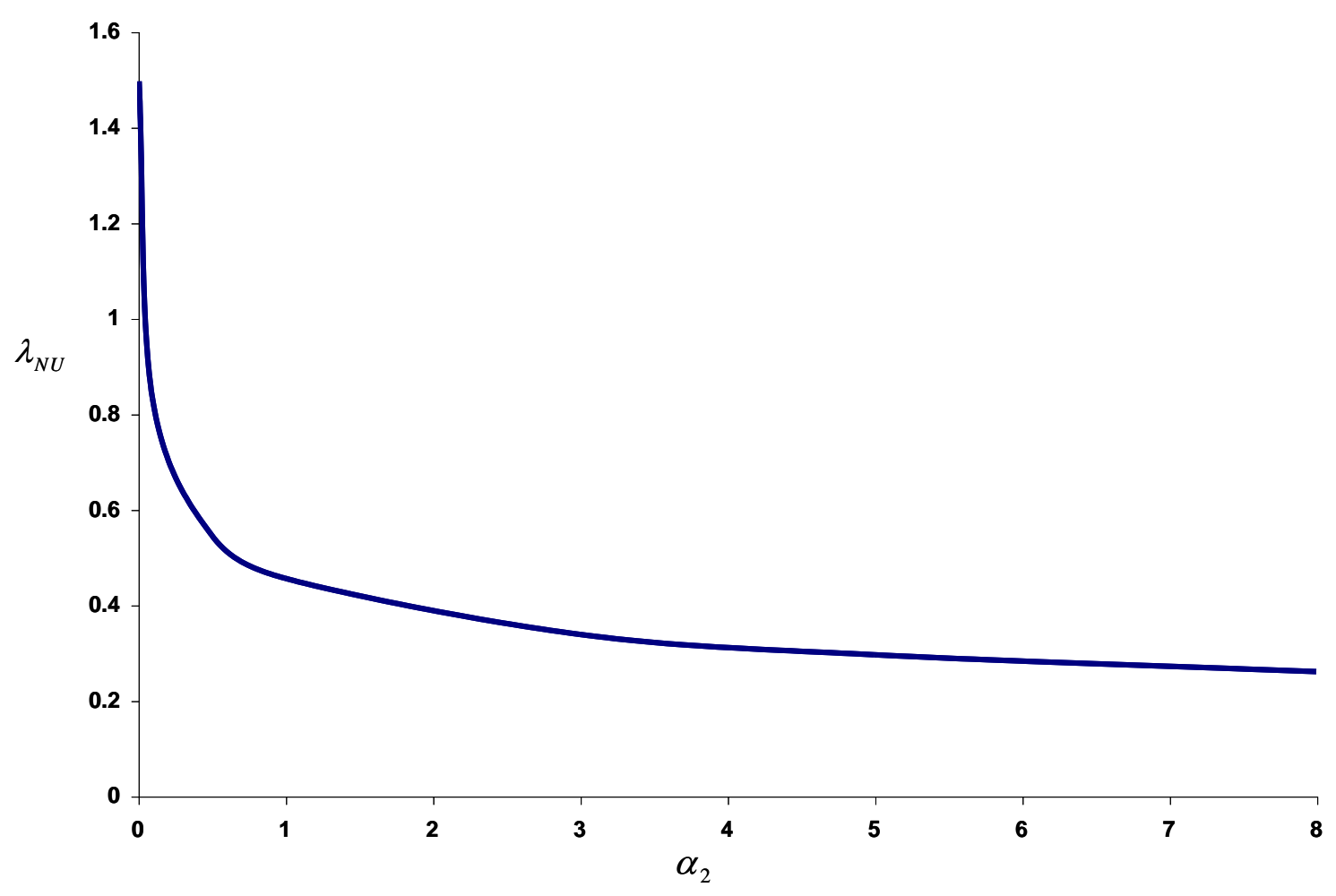

Figure 12 Dimensionless non-uniformity length scale as a function of desorption strength $H_{0}=100 \mu ; E=3.5 * 10^{-8} \mathrm{~m} / \mathrm{s} ; \mu_{0}=10^{-3} \mathrm{~kg} / \mathrm{m} . \mathrm{s} ; S=4 * 10^{-3} ; \phi_{0}=0.4$. 
(a)
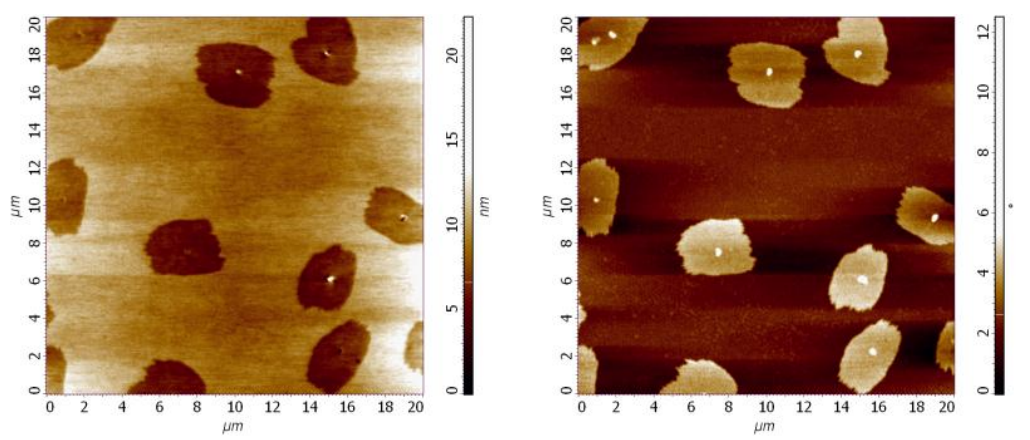

(b)

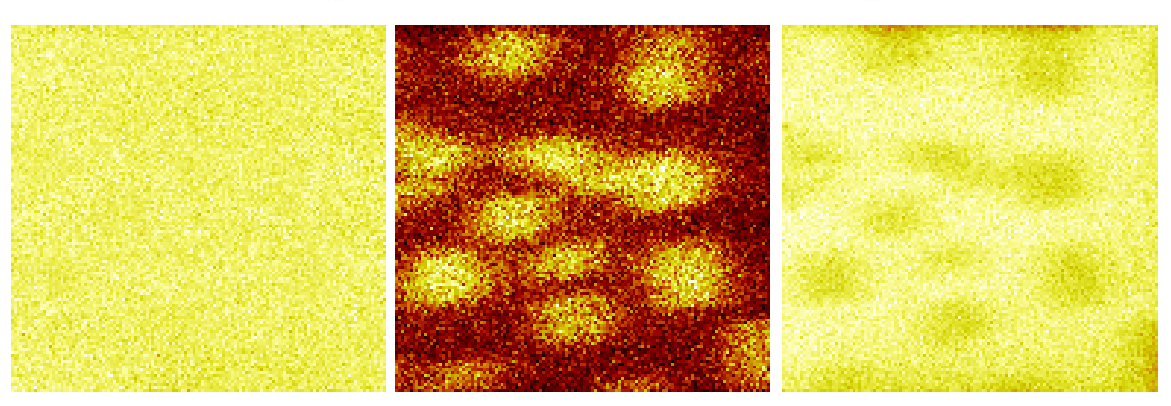

Figure 13 (a) $20 \mu \mathrm{m} \times 20 \mu \mathrm{m}$ AFM images (height on left, phase on right) of the asreceived latex with 2 wt.\% added surfactant. (b) The corresponding $17 \mu \mathrm{m}$ x17 $\mu \mathrm{m}$ ToF-SIMS maps of the same latex film, showing (left to right) $\mathrm{Na}+, \mathrm{Na}_{2} \mathrm{OH}+$ and total positive ions. Lighter color in the map indicates higher ion concentrations. 

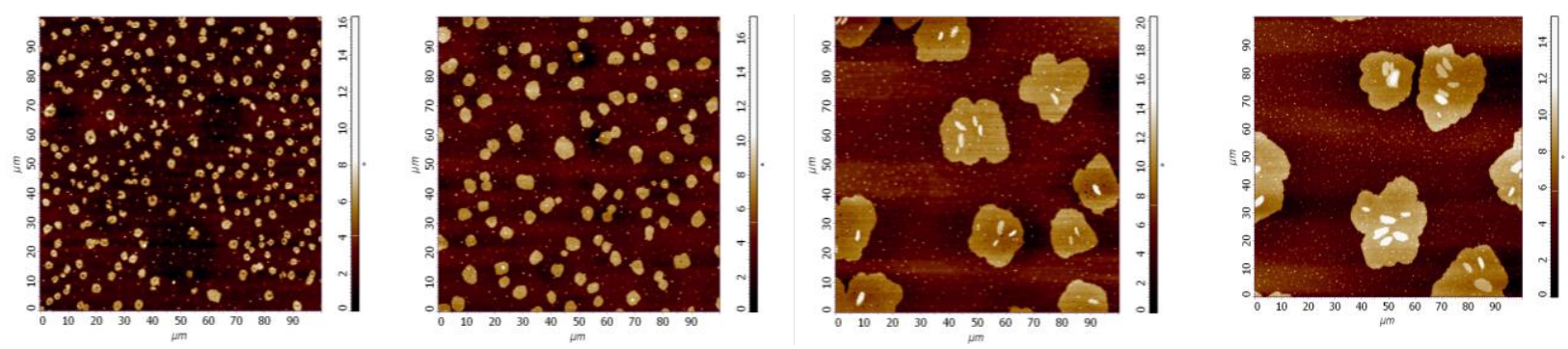

Figure 14 AFM phase images of the air surface of latex films with increasing wet thickness, from left to right: $30 \mu \mathrm{m}, 60 \mu \mathrm{m}, 90 \mu \mathrm{m}$, and $120 \mu \mathrm{m}$. All image sizes are $100 \mu \mathrm{m} \times 100 \mu \mathrm{m}$. 


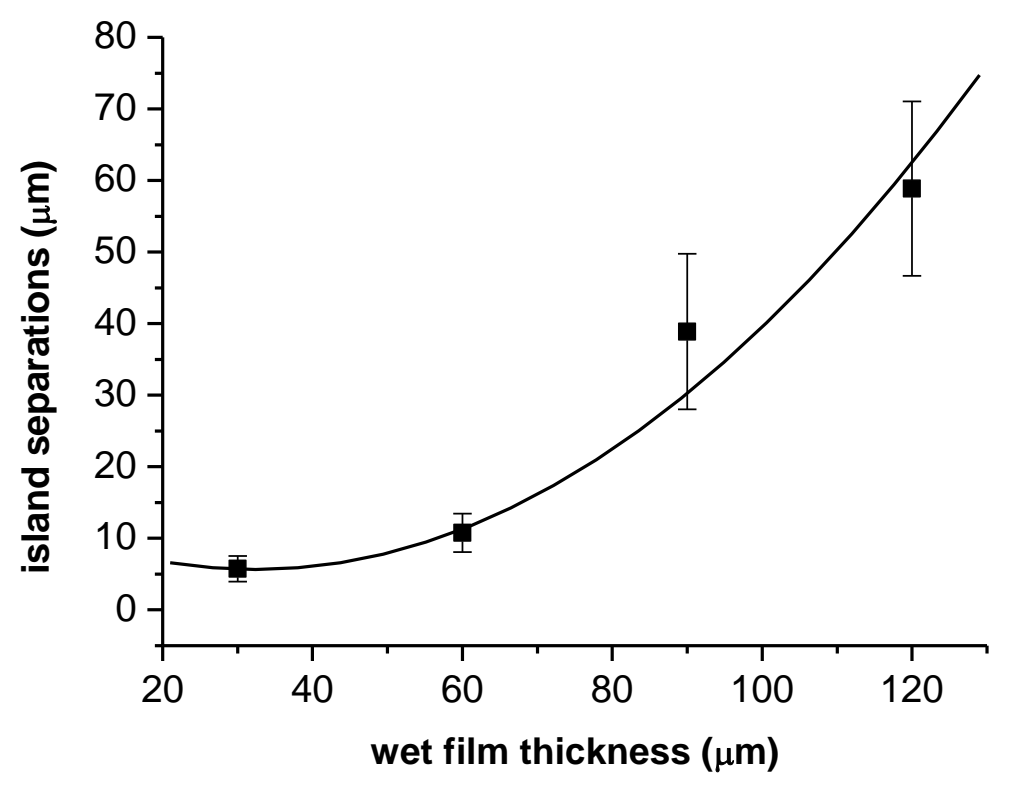

Figure 15 The dependence of surfactant "island" lateral spacing, $L_{N U}$, on wet latex film thickness, $H_{o}$, from analysis of the images in Figure 14. The solid line is a guide to the eye. 

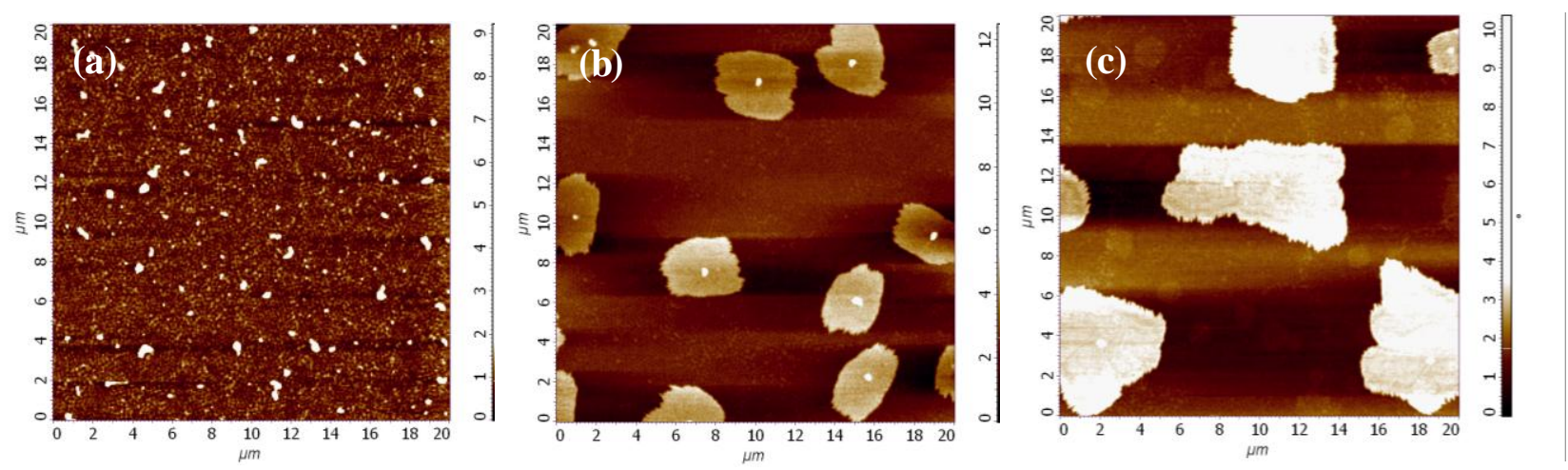

Figure 16 AFM phase images of latex films ( $40 \mu \mathrm{m}$ wet thickness) with increasing excess surfactant concentrations: (a) as-received; (b) 2 wt.\%; and (c) 4 wt.\%. Image sizes are $20 \mu \mathrm{m} \times 20 \mu \mathrm{m}$. 


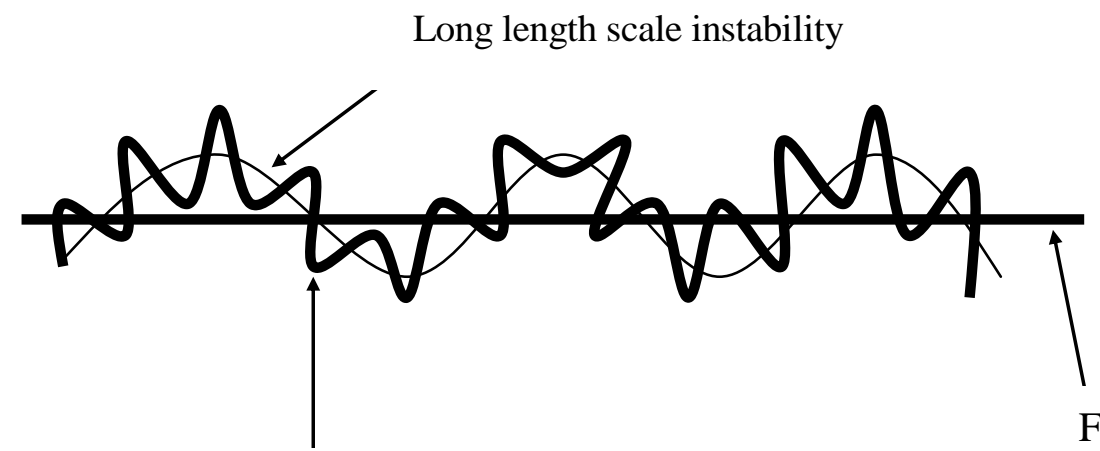

Short length scale instability

Flat, stable film

Figure 17 Sketch showing the overlap of the two instabilities. 

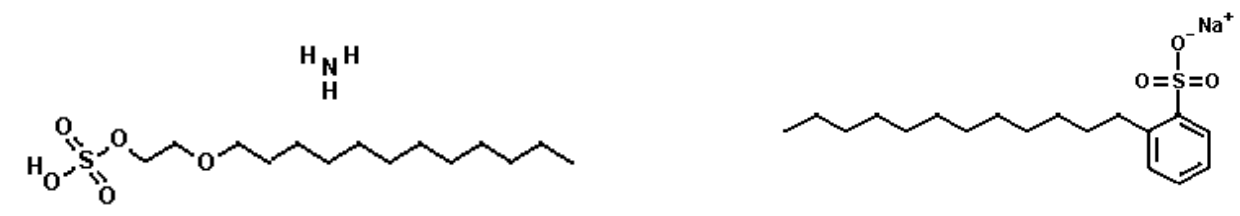

Scheme 1. Chemical structure of ALS (left) and SDBS (right).

Table 1 Two different sets of "free parameter" values with constant operating conditions using $H_{0}=50 \mu \mathrm{m} ; E=3.5 \times 10^{-8} \mathrm{~m} / \mathrm{s} ; \mu_{0}=10^{-3} \mathrm{~kg} / \mathrm{m} . \mathrm{s} ; S=4 \times 10^{-2}$

\begin{tabular}{|c|c|c|c|c|c|c|}
\hline & $\alpha_{1}$ & $\alpha$ & $\alpha_{2}$ & $P e_{S}$ & $\phi_{0}$ & $L_{0}(m)$ \\
\hline Set 1 & $\mathbf{1 0}^{-3}$ & $6.56 \times 10^{-7}$ & 1 & $2 \times 10^{4}$ & 0.4 & $\mathbf{1 . 3 7 \times 1 0 ^ { - 3 }}$ \\
\hline Set 2 & $\mathbf{1 0}^{-5}$ & $6.56 \times 10^{-5}$ & 100 & $2 \times 10^{4}$ & 0.4 & $\mathbf{1 . 3 7 \times 1 0 ^ { - 2 }}$ \\
\hline
\end{tabular}

